\section{Errors in Chromatography}

\section{Part II: Random error}

\section{R. Kaiser}

BASF M 325, D-67 Ludwigshafen/Rh.

The article in the preceding issue of the Green Pages dealing with errors stated the following:

By natural law, each measurement is subject to errors. The measuring results are called data.

Judgments or decisions on the basis of data are only possible if the following values are known:

1. the mean value of repetitive measurements $(\overline{\mathbf{x}})$,

2. the standard deviation (s),

3. the number of the repetitive measurements (n), and if mathematical statistical tests are carried out with these characteristic values.

It is only on the basis of these test results possible to arrive to an unanimous judgment or decision from the results of one measurement.

The previous article also described the calculation of the mean value (arithmetic mean) $\bar{x}$ and of the approximate standard deviation $\mathrm{s}$, and the first test treated was the Nalimov test for "runaway values".

Hereafter we are going to describe the graphic methods of statistical mathematics which permit us to obtain the following information:

First, one can evaluate whether the data show a normal statistical distribution. Only in this case it is permitted to apply the tests described below.

When checking the data for normal statistical distribution, approximate values for the mean value $\bar{x}$ and for the standard deviation $s$ are also obtained simultaneously.

As a rule, the quantitative results of chromatographic measurements correspond to a normal distribution. If graphical checking shows, that the distribution is not normal, the number of individual values must be increased by further repetitive measurements, and groups of mean values must be formed. Mean values will correspond with even greater probability to a normal distribution.

It is a widely believed misconception that statistical tests necessitate many repetitive measurements. Only three measurements are sufficient for determining an approximate standard deviation, and four to six repetitive measurements are enough to carry out relatively sophisticated statistical tests.

If the cost of a measurement is high, it is not recom. mended in chromatography to carry out more than 10 repetitive measurements.
Die gleiche Arbeit können Sie in deutscher Sprache auf Seite 220 lesen

Vous trouverez $œ$ travail en langue française en page 228
Although it is normally desirable, to have about 20 measurements testing the statistical normal distribution, it is possible to carry out a (rough) test by means of three to ten repetitive measurements.

\section{Checking the normal distribution on the basis of 3-20 individual measurements}

The following steps are necessary for the graphical approximate determination of the mean value and of the standard deviation:

1. Determine 3-20 individual values (example: five individual values obtained by repetitive measurements).

2. List the values in a table by increasing order and number them consecutively from $a=1$ to $a=n$. This consecutive numbering is necessary for the calculation of points $y_{1}$ through $y_{n}$ on the ordinate.

3. Calculate the points $y_{1}$ through $y_{n}$ for the individual values according to the following formula:

$y=50+100 \frac{a-\frac{n+1}{2}}{n}$

and list the values of $y$ in the table shown in the example.

Example :

Measured values: $37.70 ; 36.28 ; 37.30 ; 37.13$; 36.88 .

There are five results, i.e. $\mathrm{n}=5$.

List the measured values by increasing order and number them from $a=1$ through $a=n$; then calculate $y$ :

\begin{tabular}{c|l|l}
\hline Serial number & $\begin{array}{l}\text { Measured } \\
\text { values }\end{array}$ & $\begin{array}{l}\text { Points on the } \\
\text { ordinate }\end{array}$ \\
\hline $\mathrm{a}=1$ & $\mathrm{x}_{1}=36.28$ & $\mathrm{y}_{1}=10$ \\
2 & $\mathrm{x}_{2}=36.88$ & $\mathrm{y}_{2}=30$ \\
3 & $\mathrm{x}_{3}=37.13$ & $\mathrm{y}_{3}=50$ \\
4 & $\mathrm{x}_{4}=37.30$ & $\mathrm{y}_{4}=70$ \\
$5=\mathrm{n}$ & $\mathrm{x}_{5}=37.70$ & $\mathrm{y}_{5}=90$ \\
\hline
\end{tabular}

Example for calculating point $y_{2}$ corresponding to the result of the second measurement (for $a=2$, this value is $x_{2}=36.88$ ):

Formula: $\quad y_{2}=50+100 \frac{a-\frac{n+1}{2}}{n}$ 
Values: $\quad a=2 ; n=5$

Calculation: $y_{2}=50+100 \frac{2-\frac{5+1}{2}}{5}$

$$
\begin{aligned}
& =50+100 \frac{2-3}{5} \\
& =50+100\left(-\frac{1}{5}\right) \\
& =50-20 \\
y_{2} & =30
\end{aligned}
$$

4. Use a probability paper with a linear abscissa scale and with an ordinate scale corresponding to the Gaussian integral; plot the measured values $\left(\mathrm{x}_{1}\right.$, etc.) against the calculated values of $y$, etc. (see Fig. 1). In Figure 1 the abscissa is named as $\mathrm{g}$ and the ordinate as $\Sigma \%$. Connect the plotted points by a straight line. This is the so-called Hazen's line.

The value of $y=50 \%$ on the ordinate corresponds to the value of $\bar{x}$, on the abscissa which is equal to the mean value of the five individual values.

The two abscissa points corresponding to the respective ordinate points of $y=15.9 \%$ and $y=84.1 \%$ establish the limiting values of the approximate standard deviation from $-s$ to $+s$. From Fig. 1 it may be read:

$\overline{\mathrm{x}}$ is located at $\sim 37.05$

$-\mathrm{s}$ is located at 36.51

$+s$ is located at 37.60

The distance between $-s$ to $+s=2 \mathrm{~s}$ equal to $37.60-36.51=1.09$ i.e. $2 \mathrm{~s}=1.09$, or the approximate standard deviation $\mathrm{s}= \pm 0.55$.
As a control, we may calculate the values of $\bar{x}$ and $s$ according to the formulas given in the first part of this paper; the results are

$$
\begin{aligned}
\bar{x} & =37.058 \\
s & = \pm 0.53 .
\end{aligned}
$$

It is quite obvious that the method just described will lead to rather approximate data which, in turn, are again subject to errors. It is therefore desirable to use more data to check the statistical normal distribution. If twenty or more individual values are available, the following procedure can be adapted :

1. Calculate the difference between the measured highest and the lowest value.

$\begin{array}{rllll}\text { Example: } & 36.6 & 38.3 & 37.4 & 35.4 \\ 38.5 & 37.2 & 36.7 & 37.6 \\ 35.1 & 36.6 & 36.1 & 39.5 \\ 37.3 & 38.0 & 37.1 & 35.1 \\ 36.4 & 38.7 & 36.5 & 37.0\end{array}$

Highest value : $\quad 39.5$

Lowest value : $\quad 35.1$

Difference: $\quad 4.4$

2. Determine 5-6 equal ranges in which all the measured values can be included.

In the present example, the five ranges of $35.0-36.0$; $36.0-37.0 ; 37.0-38.0 ; 38.0-39.0 ; 39.0-40.0$ meet this requirement since the difference between the highest and the lowest value is $4.4 \sim 5$.

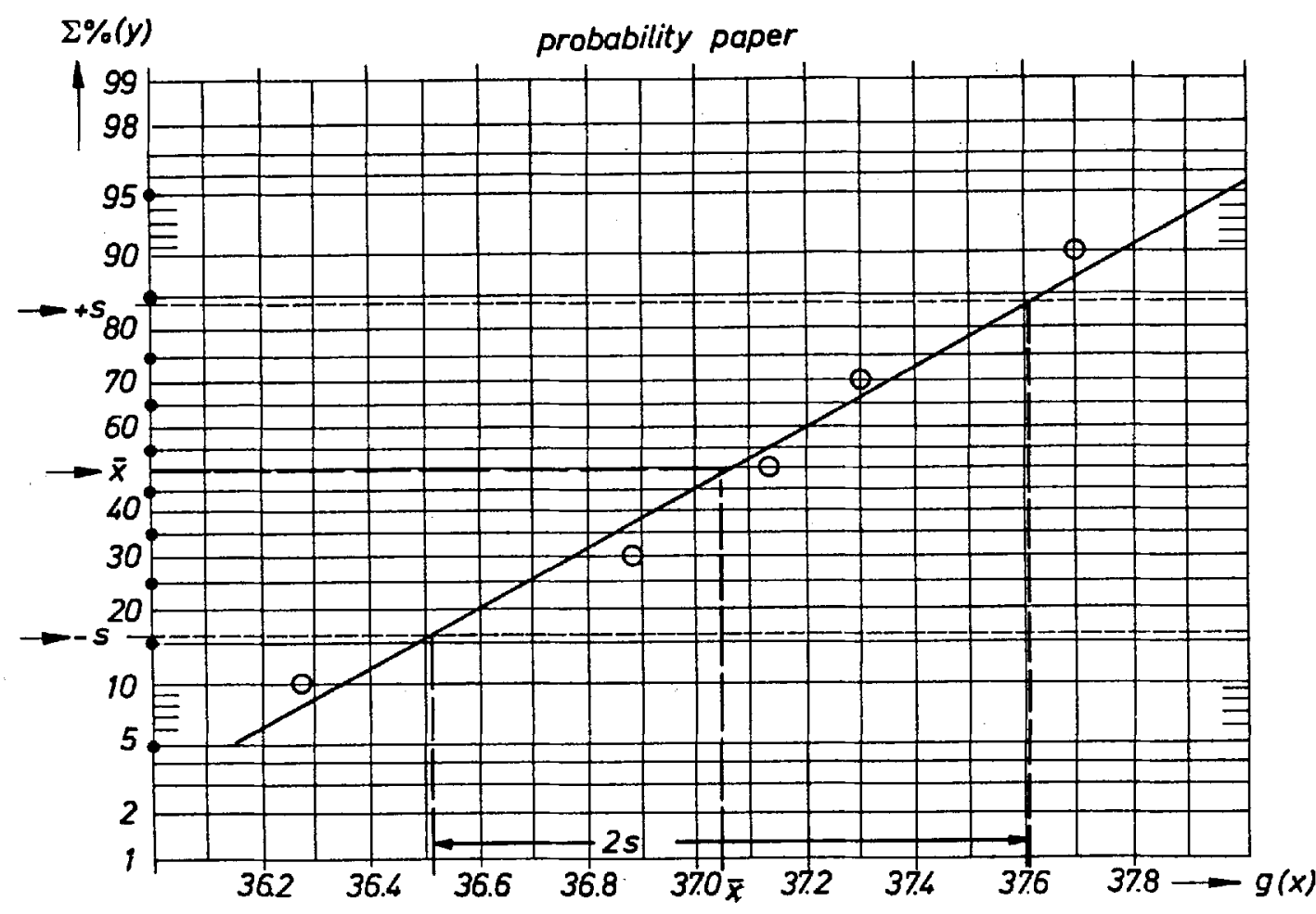

Fig. 1 
3. By means of a checklist, establish the number of the individual measured values located in each of the selected ranges; in case of the values located precisely on the limit between two ranges, divide them evenly between the lower and the higher range.

Check list :

\begin{tabular}{|c|c|c|c|}
\hline \multirow{2}{*}{ Ranges } & \multicolumn{2}{|c|}{ Number of meas. values } & \multirow{2}{*}{ Frequency } \\
\hline & & half & \\
\hline \multirow{6}{*}{$\begin{array}{l}35.0-36.0 \\
36.0-37.0 \\
37.0-38.0 \\
38.0-39.0 \\
39.0-40.0\end{array}$} & III & & 3 \\
\hline & $1 / 1 / 1 /$ & - & 6.5 \\
\hline & IIIII & -- & 6.0 \\
\hline & III & - & 3.5 \\
\hline & 1 & & 1 \\
\hline & & & $\Sigma=20.0$ \\
\hline
\end{tabular}

4. Calculate the sum of the frequency of the measured values in each range, both individually and successively, as the percent of the sum of frequencies in all ranges.

Example: There are 20 measured values.

In the range between 39.0 and 40.0 there is only one value, which is thus $5 \%$ of 20 .

\begin{tabular}{l|c|c|c}
\hline Ranges & Frequency & $\begin{array}{l}\text { Frequency } \\
\% \text { of total }\end{array}$ & $\begin{array}{l}\text { successive } \\
\text { sums as } \\
\% \text { of total } \\
(\Sigma \%)\end{array}$ \\
\hline $35.0-36.0$ & 3 & $=15.0 \%$ & $15.0 \%$ \\
$36.0-37.0$ & 6.5 & $=32.5 \%$ & $47.5 \%$ \\
$37.0-38.0$ & 6.0 & $=30.0 \%$ & $77.5 \%$ \\
$38.0-39.0$ & 3.5 & $=17.5 \%$ & $95.0 \%$ \\
$39.0-40.0$ & 1 & $=5.0 \%$ & $100.0 \%$ \\
\hline
\end{tabular}

5. Using the probability paper with a linear abscissa scale and with on ordinate scale corresponding to the Gaussian integral, plot the $\Sigma \%$ values $(15.0 \% ; 47.5 \%$; $77.5 \%$...) against the corresponding upper limiting values of the ranges (g), i.e. $x=36.0$ against $\Sigma \%=15.0$, $\mathrm{x}=37.0$ against $\Sigma \%=47.5, \mathrm{x}=38.0$ against $\Sigma \%=77.5$, etc. (see Fig. 2).

The ordinate value $\Sigma \%=50$ corresponds to the abscissa value $x=\bar{x}$, which is the mean value of the 20 individual values.

The distance between the abscissa value for $\Sigma \%=15.9 \%$ and the abscissa value for $\Sigma \%=84.1 \%$ corresponds to the range from $-s$ to $+s=2 s$.

From Fig. 2 it can be read:

$\overline{\mathbf{x}}=37.11$

$2 \mathrm{~s}$ extends from 35.96 to 38.28 , thus $2 \mathrm{~s}=2.32$, and $\mathrm{s}= \pm 1.16$.

With numerical calculation the result is $\overline{\mathrm{x}}=37.055$

$$
\mathrm{s}= \pm 1.1763
$$

The graphical check of the data material has shown that the spreading of the measured values corresponds to a normal distribution, and the relationship of the plotted points may be described quite well by a straight line; consequently the measurements supply values corresponding to a normal distribution. Thus, the following statistical tests are admissible.

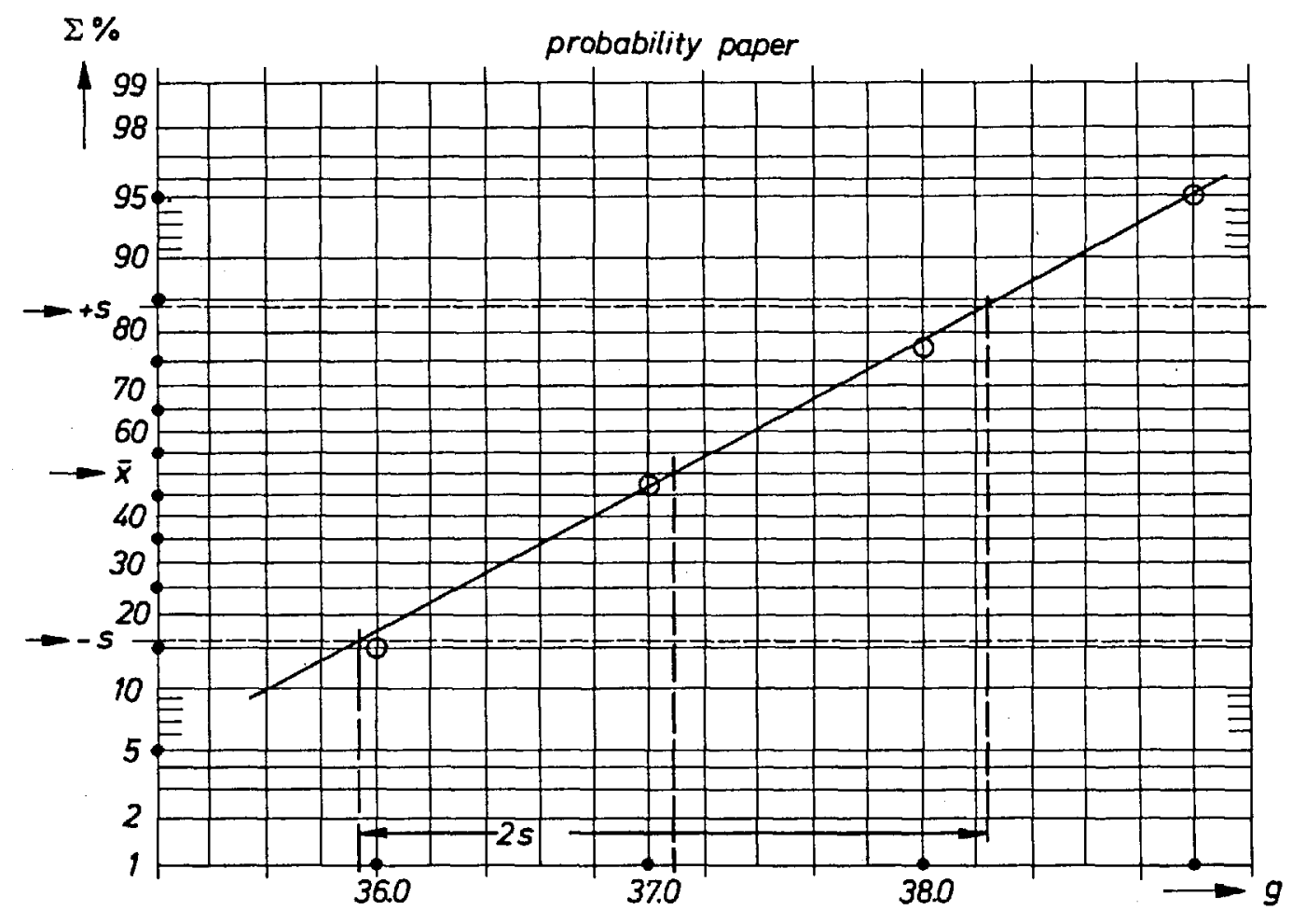

Fig. 2 


\section{t-test for the comparison of mean values}

This test permits to evaluate whether two mean values $\bar{x}_{A}$ and $\bar{x}_{B}$ are indeed different, or any given difference is not significant.

Such an evaluation on the basis of mathematic-statistical facts permits the flollowing conclusions:

a) In case if two samples have been compared, and if systematic measuring errors are excluded: the samples used for these measurements are different.

b) In case if identical samples have been analyzed in two instruments or in two separation systems: one or both instruments or separation systems produce systematic measuring errors. (This is also valid for the comparison of detectors, integrators, calibration factors, etc.)

c) In case if $\bar{x}_{A}$ and $\bar{x}_{B}$ are the results of measurements of the same product at different times or at different places: the two mean values may be combined into one, provided that the F-test for the comparison of standard deviations permits it.

A. The following data are necessary: $\bar{x}_{A}, s_{A}, n_{A}$ and $\bar{x}_{B}$, $s_{B}, n_{B}$.

B. Calculate the test value $\tau$.

$$
\begin{aligned}
& \tau=\left|\frac{\mathrm{x}_{\mathrm{A}}-\overline{\mathrm{x}}_{\mathrm{B}}}{\mathrm{s}_{\mathrm{d}}}\right| \times \sqrt{\frac{\mathrm{n}_{\mathrm{A}} \times \mathrm{n}_{\mathrm{B}}}{\mathrm{n}_{\mathrm{A}}+\mathrm{n}_{\mathrm{B}}}} \\
& \mathrm{s}_{\mathrm{d}}=\sqrt{\frac{\left(\mathrm{n}_{\mathrm{A}}-1\right) \times \mathrm{s}_{\mathrm{A}}^{2}+\left(\mathrm{n}_{\mathrm{B}}-1\right) \times \mathrm{s}_{\mathrm{B}}^{2}}{\mathrm{n}_{\mathrm{A}}+\mathrm{n}_{\mathrm{B}}-2}}
\end{aligned}
$$

C. Compare the value of $\tau$ with the statistical values $t(95)$, $t(99)$, and $t(99.9)$ which are dependent on $f=n_{A}+n_{B}-2$. The statistical values $t(95 \ldots 99.9)$ as a function of $f$ are listed in the t-Table.

If $\tau \geqslant t(95)$, then $\bar{x}_{A}$ does not differ from $\bar{x}_{B}$. Both values may be combined into one common mean value $\overline{\overline{\mathrm{X}}}$ which has the advantage of facilitating further statistical tests with better precision because now, $n$ is the sum of $n_{A}$ and $n_{B}$.

The method of calculating the common standard deviation and the total mean value of all measured values for $\bar{x}_{A}$ and $\bar{x}_{B}$ will be described in the next issue of the Green Pages.

If $t(95) \leqslant \tau<t(99): x_{A}$ differs from $x_{B}$ with $95 \%$ confidence.

If $t(99) \leqslant \tau<t(99.9): \bar{x}_{A}$ differs significantly from $\bar{x}_{B}$. If $\tau \geqslant t(99.9): \bar{x}_{A}$ differs most significantly from $\bar{x}_{B}$.

D. Numerical example :

$$
\begin{aligned}
& \mathrm{n}_{\mathrm{A}}=5 \quad \overline{\mathrm{x}}_{\mathrm{A}}=30.445 \quad \mathrm{~s}_{\mathrm{A}}= \pm 0.193 \\
& n_{B}=6 \quad \bar{x}_{B}=30.611 \quad s_{B}= \pm 0.169 \\
& s_{d}=\sqrt{\frac{4 \times 0.193^{2}+5 \times 0.169^{2}}{5+6-2}}=0.18006 \\
& \tau=\left|\frac{30.445-30.611}{0.18006}\right| \times \sqrt{\frac{5 \times 6}{5+6}}=1.522 \\
& n_{A}=5 \text { and } n_{B}=6 \text {, i.e. } f=5+6-2=9
\end{aligned}
$$

For $f=9$ the following values are listed in the $t$-Table:

$$
t(95)=2.26 \quad t(99.9)=4.78
$$

$\mathbf{t}(99)=3.25$

Decision: $\tau=1.522$ is smaller than $t(95)=2.26$, i.e the mean values $\bar{x}_{A}$ and $\bar{x}_{B}$ do not differ

The fact whether they may be combined into a new mean value must be decided by the F-test.

\section{F-test for the comparison of standard deviations}

This test permits to evaluate whether the two standard deviations $s_{A}$ and $s_{B}$ really differ from each other, or whether any given difference is not significant.

Such an evaluation based on mathematic-statistical facts permits the following conclusions:

a) In case when the data have been obtained on two in. struments, or by two measuring procedures, two methods of evaluation, or have been measured by two operators one can decide, whether these procedures, instruments, or working methods show any significant difference. It can be stated that that instrument, or working method, is better which, under otherwise identical conditions (same sample), shows the lower standard deviation.

b) If the standard deviations from two measurement series do not show any significant difference and if, as a result of the t-test (see above), the mean values also show no difference, the values obtained in both measurement series may be combined together.

A. The following data are necessary: $s_{A}, n_{A}, s_{B}$, and $n_{B}$

B. Calculate the test value $P F$.

$$
\mathrm{PF}=\left(\frac{\mathrm{s}_{\mathrm{A}}}{\mathrm{s}_{\mathrm{B}}}\right)^{2} \text { if } \mathrm{s}_{\mathrm{A}}>\mathrm{s}_{\mathrm{B}}
$$

or

$$
P F=\left(\frac{s_{B}}{s_{A}}\right)^{2} \quad \text { if } s_{B}>s_{A} \text {. }
$$

The index 1 is given to the $\mathrm{n}$ of that data group which belongs to the higher value of the standard deviation. Thus, if $s_{B}>s_{A}$, then $n_{B}=n_{1}$ and $n_{A}=n_{2}$

C. Compare the value of $\mathrm{PF}$ with the statistical values $F(95), F(99)$, and $F(99.9)$ for $f_{1}=n_{1}-1$ and $f_{2}=n_{2}-1$. The statistical values $F(95 \ldots 99.9)$ as a function of $f_{1}$ and $f_{2}$ are listed in the F-table.

If $P F \geqslant F(95)$, then $s_{A}$ does not differ from $s_{B}$. If, the $t$-test (see above), concluded that the mean values $\bar{x}_{A}$ and $\bar{x}_{B}$ do not show any significant difference, then the entire data may be combined in a statistically even more adequate group for the calculation of the common values $\overline{\overline{\mathrm{x}}}, \overline{\overline{\mathrm{s}}}, \overline{\overline{\mathrm{n}}}$. This will be described in the next issue of the Green Pages.

If $F(95) \leqslant P F<F(99): s_{A}$ differs from $s_{B}$ with $95 \%$ confidence.

If $F(99) \leqslant P F<F(99.9): s_{A}$ differs significantly from $s_{B}$.

If $P F \geqslant F(99.9): s_{A}$ differs most significantly from $s_{B}$ 


\section{Numerical example:}

$$
\begin{array}{rlrl}
\mathrm{n}_{\mathrm{A}} & =5 & \mathrm{~s}_{\mathrm{A}}=0.2525 \\
\mathrm{n}_{\mathrm{B}}=5 & \mathrm{~s}_{\mathrm{B}}=0.0856 \\
\mathrm{PF} & =\left(\frac{0.2525}{0.0856}\right)^{2}=8.701 \\
\mathrm{n}_{\mathrm{A}}=\mathrm{n}_{1} \text { because } \mathrm{s}_{\mathrm{A}}>\mathrm{s}_{\mathrm{B}} \\
\mathrm{f}_{1}=\mathrm{n}_{\mathrm{A}}-1, & \mathrm{f}_{2}=\mathrm{n}_{\mathrm{B}}-1 \\
\mathrm{f}_{1}=3, & \mathrm{f}_{2}=4
\end{array}
$$

For these values of $f_{1}$ and $f_{2}, \begin{array}{r}F(95)=6.59 \\ F(99)=16.69 \\ F(99.9)=56.18\end{array}$

This means that $\mathrm{F}(99)>\mathrm{PF}=8.701>\mathrm{F}(95)$; consequently, $s_{A}$ is probably higher than $s_{B}$. If this result with a $95 \%$ confidence level is not satisfactory, more measurements should be carried out. The F-test is not particularly accurate if too few repetitive measurements are available.

t. values see page 224

F-values see pages $225,226,227$ 


\section{Fehler in der Chromatographie}

\section{Teil II: Zufallsfehler}

\section{R. Kaiser}

BASF M 325, D-67 Ludwigshafen/Rh.
You can read this paper in English language on page 215

Vous trouverez ce travail en langue française en page 228
In der vorangegangenen Folge der grünen Seiten über Fehler wurde festgestellt:

Jede Messung ist naturgesetzlich mit Fehlern behaftet. Die Meßergebnisse werden Daten genannt.

Urteile und Entscheidungen auf Grund von Daten sind nur möglich, wenn man

1. den Mittelwert $\overline{\mathbf{x}}$ von wiederholten Messungen,

2. die Standardabweichung s,

3. die Zahl $\mathbf{n}$ der wiederholten Messungen

kennt und mit diesen Kenngrößen mathematisch statistische Tests ausführt.

Erst die Ergebnisse dieser Tests liefern die Grundlage, auf welcher ein Urteil oder eine Entscheidung aus den Werten einer Messung naturwissenschaftlich einwandfrei möglich ist.

Es wurde die Berechnung des Mittelwertes (arithmetisches Mit tel) $\overline{\mathrm{x}}$ und der Näherungsstandardabweichung $s$ angegeben. Als erster Test wurde der Nalimov-Test auf „Ausreißer" behandelt.

Im folgenden sollen graphische Methoden der statistischen Mathematik beschrieben werden, welche folgende Informationen liefern:

Man erkennt, ob die Daten statistisch normalverteilt sind. Nur dann ist es erlaubt, die im folgenden beschriebenen Tests anzuwenden.

Bei der Prüfung auf Normalverteilung erhält man zugleich Näherungswerte fuir den Mittelwert $\bar{x}$ und die Standardabweichung s.

In der Regel sind die quantitativen Resultate chromatographischer Messungen normalverteilt. Ergibt die graphische Prüfung, daß keine Normalverteilung vorliegt, dann muß man durch wiederholte Messungen die Zahl der Einzelwerte erhöhen und Gruppen von Mittelwerten bilden. Mittelwerte sind mit noch größerer Wahrscheinlichkeit normalverteilt.

Es ist ein weitverbreiteter Irrtum, daß man für statistische Tests sehr viele wiederholte Messungen benötigt.

Schon aus drei Messungen läßt sich eine Näherungsstandardabweichung ermitteln.

Schon vier bis sechs wiederholte Messungen erlauben relativ scharfe statistische Tests.

Mehr als zehn Wiederholmessungen zu machen, ist, wenn die Kosten der Einzelmessung groß sind, in der Chromatographie nicht zu empfehlen.
Es ist sogar möglich, mit drei bis zehn Wiederholmessungen eine (grobe) Prüfung auf statistische Normalverteilung durchzuführen. Normalerweise sind aber etwa zwanzig Messungen erwünscht.

\section{Prüfung auf Normalverteilung bei Vorliegen von 3 bis 20 Einzelmessungen}

Graphische Näherungsbestimmung des Mittelwertes und der Stand ardabweichung

1. Bestimme 3 bis 20 Einzelwerte (Beispiel: 5 Einzelwerte durch wiederholte Messung).

2. Ordne die Werte nach ihrer Größe in einer Tabelle und bezeichne sie fortlaufend mit $a=1$ bis $a=n$. Diese fortlaufende Bezeichnung wird benötigt, um nach der folgenden Formel die Ordinatenpunkte $y_{1}$ bis $y_{n}$ berechnen zu können.

3. Berechne die Ordinatenpunkte $y_{1}$ bis $y_{n}$ für die einzelnen Meßwerte nach der Formel

$y=50+100 \frac{a-\frac{n+1}{2}}{n}$

und trage die Werte für $\mathrm{y}$ in die folgende Tabelle ein:

Beispiel:

Meßwerte: $37.70 ; 36.28 ; 37.30 ; 37.13 ; 36.88$.

Es liegen 5 Meßwerte vor, also $\mathrm{n}=5$.

Ordne die Meßwerte nach ansteigendem Wert und lege deren Kennzeichnung $\mathrm{a}=1$ bis $\mathrm{a}=\mathrm{n}$ fest; berechne y:

\begin{tabular}{c|c|c}
\hline Kennzeichnung & Meßwert & Ordinatenpunkte \\
\hline $\mathrm{a}=1$ & 36.28 & $\mathrm{y}_{1}=10$ \\
2 & 36.88 & $\mathrm{y}_{2}=30$ \\
3 & 37.13 & $\mathrm{y}_{3}=50$ \\
4 & 37.30 & $\mathrm{y}_{4}=70$ \\
$5=\mathrm{n}$ & 37.70 & $\mathrm{y}_{5}=90$ \\
\hline
\end{tabular}

Beispiel für die Berechnung des Ordinatenpunktes $y_{2}$ für den 2. Meßwert (zu a $=2$ lautet der Meßwert 36.88):

Formel:

$y_{2}=50+100 \frac{a-\frac{n+1}{2}}{n}$ 
Werte:

$\mathrm{a}=2 ; \mathrm{n}=5$

Berechnung:

$$
\begin{aligned}
y_{2} & =50+100 \frac{2-\frac{5+1}{2}}{5} \\
& =50+100 \frac{2-3}{5} \\
& =50+100\left(-\frac{1}{5}\right) \\
& =50-20 \\
y_{2} & =30
\end{aligned}
$$

4. Beschaffe Wahrscheinlichkeitspapier, dessen $x$-Achse linear und dessen y-Achse nach dem Gauß'schen Integral geteilt ist, und trage bei den berechneten Werten für y die Größe der zugehörigen Meßwerte ein, siehe Bild 1. Die $x$-Achse ist dort mit $g$ bezeichnet, die $\mathrm{y}$-Achse mit $\Sigma \%$. Verbinde die $\mathrm{x}, \mathrm{y}$-Punkte mit einer Geraden. Diese heißt Hazen'sche Gerade.

Zum Ordinatenwert y $=50 \%$ gehört der Abszissenwert $\overline{\mathbf{x}}$, das ist der Mittelwert der 5 Einzelwerte.

$\mathrm{Zu}$ den Ordinatenpunkten $\mathrm{y}=15.9 \%$ und $\mathrm{y}=84.1 \%$ gehören 2 Abszissenwerte, welche die Grenzwerte für die Näherungsstandardabweichung von $-s$ bis $+s$ einschließen. Man kann in Bild 1 ablesen:

$\bar{x}$ liegt bei $\sim 37.05$

-s liegt bei 36.51

$+s$ liegt bei 37.60

Der Wert von $-s$ bis $+s=2 s$ reicht von $x=36.51$ bis $x=37.60$, also beträgt $2 \mathrm{~s}=1.09$ oder die Näherungsstandardabweichung $\pm 1 \mathrm{~s}$ beträgt $\sim 0.55$.

Rechnet man $x$ und $s$ numerisch nach den in der ersten Folge dieser grünen Seiten angegebenen Formeln aus, so erhält man

$\overline{\mathrm{x}}=37.058$

$\mathrm{s}= \pm 0.53$

Es ist ganz klar, daß die soeben beschriebene Methode zu recht groben Daten führt, die ihrerseits wiederum fehlerbehaftet sind. Es ist daher wünschenswert, mehr Daten zur Prüfung auf statistische Normalverteilung einzusetzen. Hat man 20 und mehr Einzelmeßwerte, dann geht man wie folgt vor:

1. Stelle die Differenz zwischen dem größten und kleinsten Meßwert fest.

$\begin{array}{lllll}\text { Beispiel: } & 36.6 & 38.3 & 37.4 & 35.4 \\ & 38.5 & 37.2 & 36.7 & 37.6 \\ & 35.1 & 36.6 & 36.1 & 39.5 \\ & 37.3 & 38.0 & 37.1 & 35.1 \\ & 36.4 & 38.7 & 36.5 & 37.0\end{array}$

Größter Wert: 39.5 Differenz:

Kleinster Wert: $\quad 35.1$
4.4

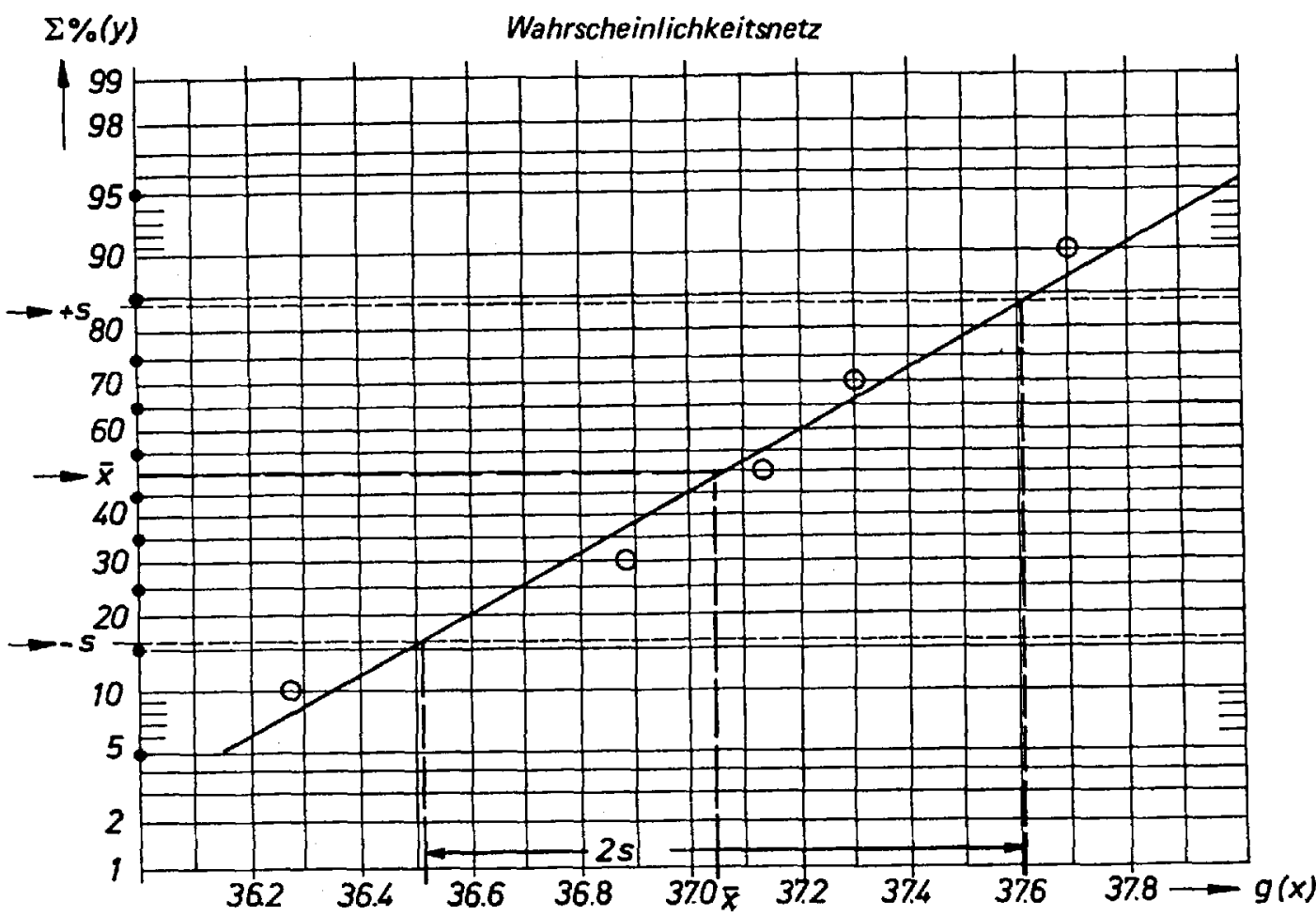

Fig. 1 


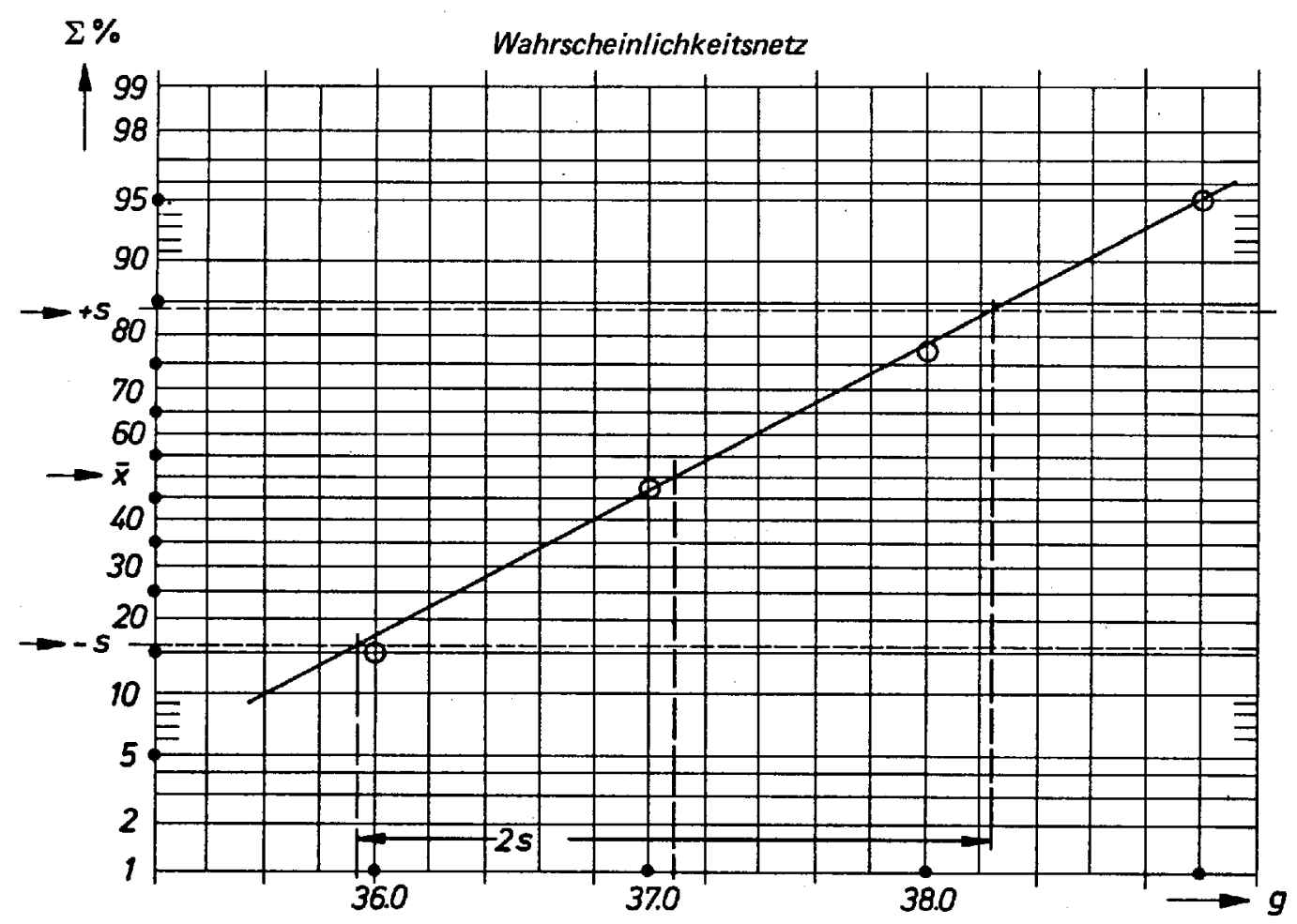

Fig. 2

2. Bestimme 5 bis 6 gleich große Meßwertbereiche, in denen alle Einzelmeßwerte liegen.

Im vorliegenden Beispiel ergibt sich, daß 5 Meßwertbereiche von $35.0-36.0 ; 36.0-37.0 ; 37.0-38.0$; $38.0-39.0 ; 39.0-40.0$ die Forderung von Punkt 2 erfüllen, da die Differenz zwischen größtem und kleinstem Wert $4.4 \sim 5$ beträgt.

3. Stelle in Form einer Strichliste fest, wieviele Einzelmeßwerte in den jeweiligen Meßbereich fallen, zähle die genau auf die Grenze von 2 Bereichen fallenden Meßwerte je zur Hälfte dem kleineren und größeren Bereich zu.

Strichliste:

\begin{tabular}{|c|c|c|c|}
\hline Bereiche & \multicolumn{2}{|c|}{ Anzahl Meßwerte } & Häufigkeit*) \\
\hline $\begin{array}{l}35.0-36.0 \\
36.0-37.0 \\
37.0-38.0 \\
38.0-39.0 \\
39.0-40.0\end{array}$ & $\begin{array}{l}111 \\
111111 \\
11111 \\
111 \\
1\end{array}$ & $\begin{array}{l}- \\
-- \\
-\end{array}$ & $\begin{array}{l}3 \\
6.5 \\
6.0 \\
3.5 \\
1 \\
\end{array}$ \\
\hline & & & $\Sigma=20.0$ \\
\hline
\end{tabular}

4. Berechne die Summe der Häufigkeit der in die Meßwertbereiche fallenden Werte einzeln und fortlaufend in Prozent.

Beispiel: Es lagen 20 Meßwerte vor. Im Bereich $39.0-40.0$ befindet sich $1 \mathrm{MeB}$ wert, das sind $5 \%$ von 20 Werten.

*) Häufigkeit: Dies ist hier die Summe der ganzen und halben Striche in der Strichliste

\begin{tabular}{l|c|l|c}
\hline Bereiche & Häufigkeit & Häufigkeit \% & $\begin{array}{l}\text { fortlaufende } \\
\text { Summen } \% \\
(\Sigma \%)\end{array}$ \\
\hline $35.0-36.0$ & 3 & $=15.0 \%$ & $15.0 \%$ \\
$36.0-37.0$ & 6.5 & $=32.5 \%$ & $47.5 \%$ \\
$37.0-38.0$ & 6.0 & $=30.0 \%$ & $77.5 \%$ \\
$38.0-39.0$ & 3.5 & $=17.5 \%$ & $95.0 \%$ \\
$39.0-40.0$ & 1 & $=5.0 \%$ & $100.0 \%$ \\
\hline
\end{tabular}

5. Trage in Wahrscheinlichkeitspapier, dessen $x$-Achse linear und dessen y-Achse nach dem Gauß'schen Integral geteilt ist, zu den $\Sigma \%$-Werten $(15.0 \%, 47.5 \%$, $77.5 \%$...) die zugehörigen oberen Grenzwerte der Meßwertbereiche (g) ein, also $\mathrm{zu} x=36.0$ den Wert $\Sigma \%=15.0, \mathrm{zu} \mathrm{x}=37.0$ den Wert $\Sigma \%=47.5, \mathrm{zu}$ $\mathrm{x}=38.0$ den Wert $\Sigma \%=77.5$ usw., siehe Bild 2 .

$\mathrm{Zu} \Sigma \%=50$ gehört der Abszissenwert $\mathrm{x}=\overline{\mathrm{x}}$, das ist der Mittelwert der 20 Einzelwerte.

Zwischen dem Abszissenwert zu $\Sigma \%=15.9 \%$ und dem Abszissenwert zu $\Sigma \%=84.1 \%$ liegt der Bereich von $-\mathrm{s}$ bis $+\mathrm{s}=2 \mathrm{~s}$.

Aus Bild 2 liest man ab:

$\overline{\mathrm{x}}=37.11$

$2 \mathrm{~s}$ reicht von 35.96 bis 38.28 , d.h. $2 \mathrm{~s}=2.32$, also $s= \pm 1.16$

Numerisch berechnet findet man:

$\overline{\mathrm{x}}=37.055$

$s= \pm 1.1763$

Die graphische Prüfung des Zahlenmaterials hat ergeben: Die Streuung der Meßwerte ist normalverteilt, der Verlauf der Meßpunkte wird recht gut durch eine Gerade beschrie. ben. 
Demzufolge liefert das Meßverfahren normalverteilte Meßwerte, also sind die folgenden statistischen Tests zulässig.

\section{t-Test zum Vergleich von Mittelwerten}

Dieser Test erlaubt das Urteil, ob sich 2 Mittelwerte $\overline{\mathbf{x}}_{\mathbf{A}}$ und $\overline{\mathbf{x}}_{\mathbf{B}}$ voneinander wirklich unterscheiden oder ob ein Unterschied nicht signifikant ist.

Ein solches mathematisch-statistisch gesichertes Urteil erlaubt folgende Aussagen:

a) Wenn 2 Proben verglichen wurden und systematische Meßfehler ausgeschlossen sind: Die den Messungen zugrundeliegenden Proben sind unterschiedlich.

b) Wenn exakt gleiche Proben auf 2 Geräten oder in 2 Trennsystemen untersucht wurden: Eines oder beide Geräte bzw. Trennsysteme erzeugen systematische Meßfehler. (Gilt auch für den Vergleich von Detektoren, Integratoren, Eichfaktoren usw.)

c) Wenn $\overline{\mathbf{x}}_{\mathbf{A}}$ und $\overline{\mathbf{x}}_{\mathbf{B}}$ das Ergebnis von Messungen am gleichen Produkt zu unterschiedlichen Zeiten oder an unterschiedlichen Orten sind: Die beiden Mittelwerte dürfen zusammengefaßt werden, vorausgesetzt, der FTest zum Vergleich von Stand ardabweichungen läßt dies zu.

A. Man benötigt die Daten $\bar{x}_{A}, s_{A}, n_{A}$ und $\bar{x}_{B}, s_{B}, n_{B}$.

B. Man berechnet die Prüfgröße TAU.

$$
\begin{aligned}
& \mathrm{TAU}=\frac{\bar{x}_{A}-\bar{x}_{B}}{s_{d}} \times \sqrt{\frac{n_{A} \times n_{B}}{n_{A}+n_{B}}} \\
& s_{d}=\sqrt{\frac{\left(n_{A}-1\right) \times s_{A}^{2}+\left(n_{B}-1\right) \times s_{B}^{2}}{n_{A}+n_{B}-2}}
\end{aligned}
$$

C. Man entscheidet durch Vergleich von TAU mit den statistischen Werten $t(95), t(99)$ und $t(99.9)$, die von $f=n_{A}+n_{B}-2$ abhängen. Die statischen Werte $t(95 \ldots 99.9)$ in Abhängigkeit von $f$ findet man in der t-Tabelle, siehe unten.

Wenn TAU $\geqslant t(95)$, dann unterscheidet sich $\bar{x}_{A}$ von $\bar{x}_{B}$ nicht. Man darf beide Werte zu einem Gesamtmittelwert $\overline{\mathrm{x}}$ zusammenfassen, was den Vorteil hat, daß weitere statistische Tests mit größerer Schärfe möglich sind, denn $n$ ist dann die Summe von $n_{A}$ und $n_{B}$. Wie gemeinsame Standardabweichung und der Gesamtmittelwert aller Meßwerte $z u \overline{\mathbf{x}}_{\mathrm{A}}$ und $\overline{\mathbf{x}}_{\mathrm{B}}$ berechnet werden, wird in der nächsten Folge der grünen Seiten angegeben.

Wenn $t(95) \leqslant$ TAU $<t(99): \bar{x}_{\mathbf{A}}$ unterscheidet sich mit $95 \%$ Sicherheit von $\bar{x}_{\mathrm{B}}$.

Wenn $t(99) \leqslant T A U<t(99.9): \bar{x}_{A}$ unterscheidet sich signigikant von $\overline{\mathrm{X}}_{\mathrm{B}}$.

Wens $T A U \geqslant t(99.9): \bar{x}_{A}$ unterscheidet sich hochsignifikant von $\overline{\mathbf{x}}_{\mathrm{B}}$.
D. Zahlenbeispiel:

$$
\begin{aligned}
& \mathrm{n}_{\mathrm{A}}=5 \quad \overline{\mathrm{x}}_{\mathrm{A}}=30.445 \quad \mathrm{~s}_{\mathrm{A}}= \pm 0.193 \\
& \mathrm{n}_{\mathrm{B}}=6 \quad \overline{\mathrm{x}}_{\mathrm{B}}=30.611 \quad \mathrm{~s}_{\mathrm{B}}= \pm 0.169 \\
& s_{d}=\sqrt{\frac{4 \times 0.193^{2}+5 \times 0.169^{2}}{5+6-2}}=0.18006 \\
& \mathrm{TAU}=\left|\frac{30.445-30.611}{0.18006}\right| \times \sqrt{\frac{5 \times 6}{5+6}}=1.522 \\
& n_{\Lambda}=5 \text { und } n_{B}=6 \text {, also } f=5+6-2=9 \\
& \mathrm{Zu} \mathrm{f}=9 \text { findet man in der } \mathrm{t} \text {-Tabelle: } \mathrm{t}(95)=2.26 \\
& \mathrm{t}(99)=3.25 \\
& t(99.9)=4.78
\end{aligned}
$$

Entscheid: $\mathrm{TAU}=1.522$ ist kleiner als $t(95)=2.26$, also unterscheiden sich die Mittelwerte $\overline{\mathbf{x}}_{\mathrm{A}}$ und $\overline{\mathbf{x}}_{\mathrm{B}}$ nicht.

Ob man sie zu einem neuen Mittelwert zusammenfassen darf, entscheidet der F-Test.

\section{F-Test zum Vergleich von Standardabweichungen}

Dieser Test erlaubt das Urteil, ob sich 2 Standardabweichungen $s_{A}$ und $s_{B}$ voneinander wirklich unterscheiden oder ob ein Unterschied nicht signifikant ist.

Ein solches mathematisch-statistisch gesichertes Urteil erlaubt folgende Aussagen:

a) Wenn 2 Instrumente, 2 Meßverfahren, 2 Auswertemethoden oder Werte von 2 Bearbeitern die Daten liefferten, ob sich die Verfahren, Instrumente oder Arbeitsweisen signifikant unterscheiden. Das bessere Verfahren oder Instrument oder die bessere Arbeitsweise ist jene, welche unter sonst gleichen Bedingungen (gleiche Probe) die kleinere Standardabweichung lieferte.

b) Wenn sich aus 2 MeBreihen die Standardabweichungen nicht signifikant unterscheiden und als Ergebnis des t-Tests (siehe oben) auch die Mittelwerte nicht unterscheiden, dürfen alle Daten beider Meßreihen zu Gesamtdaten zusammengefaßt werden.

A. Man benötigt die Daten $s_{A}, n_{A}, s_{B}$ und $n_{B}$.

B. Man berechnet die Prüfgröße PF.

$\mathrm{PF}=\left(\frac{s_{A}}{s_{B}}\right)^{2}$, wenn $s_{A}>s_{B}$
oder
$P F=\left(\frac{s_{B}}{s_{A}}\right)^{2}$, wenn $s_{B}>s_{A}$.

Das n jener Datengruppe bekommt den Index 1 , welches zum größeren Wert der Standardabweichung gehört. Wenn also $s_{B}>s_{A}$, dann heißt $n_{B}=n_{1}$ und $\mathrm{n}_{\mathrm{A}}=\mathrm{n}_{\mathbf{2}}$. 
C. Man entscheidet durch Vergleich von PF mit den statistischen Werten $F(95), F(99)$ und $F(99.9)$ für $f_{1}=n_{1}-1$ und $f_{2}=n_{2}-1$. Die statistischen Werte $F(95 \ldots 99.9)$ in Abhängigkeit von $f_{1}$ und $f_{2}$ findet man in der F-Tabelle.

Wenn $P F \geqslant F(95)$, dann unterscheidet sich $s_{A}$ von $s_{B}$ nicht. Man darf, wenn sich nach dem t-Test (siehe oben) auch die Mittelwerte $\bar{x}_{A}$ und $\bar{x}_{B}$ nicht signifikant unterscheiden, aus dem gesamten Datenmaterial eine neue statistisch noch schlagfähigere gemeinsame Datengruppe $\overline{\overline{\mathrm{x}}}, \overline{\overline{\mathrm{s}}}, \overline{\overline{\mathrm{n}}}$ berechnen. Dies wird in der nächsten Folge der grünen Seiten beschrieben.

Wenn $F(95) \leqslant P F<F(99) ; s_{A}$ unterscheidet sich mit $95 \%$ Sicherheit von $\mathrm{s}_{\mathrm{B}}$.

Wenn $F(99) \leqslant P F<F(99.9): s_{A}$ unterscheidet sich signifikant von $\mathrm{s}_{\mathrm{B}}$.

Wenn $P F \geqslant F(99.9): s_{A}$ unterscheidet sich hochsignifikant von $\mathrm{s}_{\mathrm{B}}$.

D. Zahlenbeispiel:

$n_{A}=4 \quad s_{A}=0.2525$

$\mathrm{n}_{\mathrm{B}}=5 \quad \mathrm{~s}_{\mathrm{B}}=0.0856$

$\mathrm{PF}=\left(\frac{0.2525}{0.0856}\right)^{2}=8.701$

$\mathrm{n}_{\mathrm{A}}=\mathrm{n}_{1}$, weil $\mathrm{s}_{\mathrm{A}}>\mathrm{s}_{\mathrm{B}}$

$\mathrm{f}_{1}=\mathrm{n}_{\mathrm{A}}-1, \mathrm{f}_{2}=\mathrm{n}_{\mathrm{B}}-1$

$f_{1}=3 \quad, f_{2}=4$

Für diese Werte von $f_{1}$ und $f_{2}$ ist $F(95)=6.59$

$$
\mathrm{F}(99)=16.69
$$$$
F(99.9)=56.18
$$

Also: $F(99)>P F=8.701>F(95)$, demnach ist $s_{A}$ wahrscheinlich größer als $s_{B}$.

Wenn man mit diesem nur auf $95 \%$ sicheren Ergebnis nicht zufrieden ist, sollte man mehr Messungen durchführen. Der F-Test ist nicht besonders scharf, wenn zu wenige Wiederholmessungen vorliegen.

\begin{tabular}{|c|c|c|c|}
\hline \multicolumn{4}{|c|}{ t - Tabelle } \\
\hline f & $t(95)$ & $t(99)$ & $t(99.9)$ \\
\hline 1 & 12.71 & 63.66 & 636.62 \\
\hline 2 & 4.30 & 9.92 & 31.60 \\
\hline 3 & 3.18 & 5.84 & 12.92 \\
\hline 4 & 2.78 & 4.60 & 8.61 \\
\hline 5 & 2.57 & 4.03 & 6.86 \\
\hline 6 & 2.45 & 3.71 & 5.96 \\
\hline 7 & 2.37 & 3.50 & 5.41 \\
\hline 8 & 2.31 & 3.36 & 5.04 \\
\hline 9 & 2.26 & 3.25 & 4.78 \\
\hline 10 & 2.23 & 3.17 & 4.59 \\
\hline 11 & 2.20 & 3.11 & 4.44 \\
\hline 12 & 2.18 & 3.06 & 4.32 \\
\hline 13 & 2.16 & 3.01 & 4.22 \\
\hline 14 & 2.15 & 2.98 & 4.14 \\
\hline 15 & 2.13 & 2.95 & 4.07 \\
\hline 16 & 2.12 & 2.92 & 4.02 \\
\hline 17 & 2.11 & 2.90 & 3.96 \\
\hline 18 & 2.10 & 2.88 & 3.92 \\
\hline 19 & 2.09 & 2.86 & 3.88 \\
\hline 20 & 2.08 & 2.85 & 3.85 \\
\hline 25 & 2.060 & 2.787 & 3.725 \\
\hline 30 & 2.042 & 2.750 & 3.646 \\
\hline 35 & 2.030 & 2.724 & 3.592 \\
\hline 40 & 2.021 & 2.704 & 3.551 \\
\hline 45 & 2.014 & 2.689 & 3.521 \\
\hline 50 & 2.009 & 2.678 & 3.496 \\
\hline 100 & 1.984 & 2.626 & 3.390 \\
\hline 200 & 1.972 & 2.601 & 3.340 \\
\hline 300 & 1.969 & 2.595 & 3.328 \\
\hline 400 & 1.967 & 2.590 & 3.318 \\
\hline 500 & 1.965 & 2.586 & 3.310 \\
\hline 600 & 1.964 & 2.585 & 3.307 \\
\hline 700 & 1.963 & 2.584 & 3.304 \\
\hline 800 & 1.963 & 2.583 & 3.302 \\
\hline$\infty$ & 1.960 & 2.576 & 3.291 \\
\hline
\end{tabular}




\begin{tabular}{|c|c|c|c|c|c|}
\hline 8 & 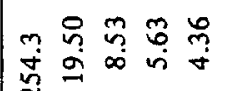 & 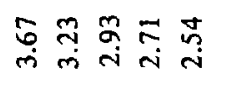 & 导兽 $\vec{i} \underset{i}{\stackrel{i}{i}}$ & 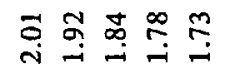 & 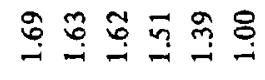 \\
\hline$\stackrel{\sim}{\Delta}$ & 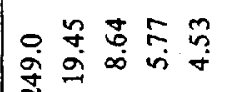 & 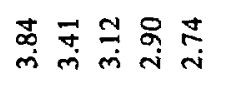 & 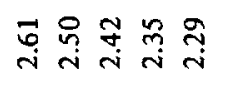 & 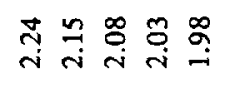 & 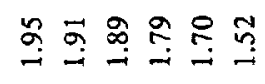 \\
\hline$\tilde{\lambda}$ & 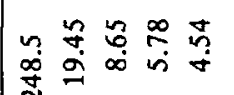 & 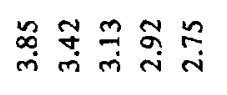 & $\stackrel{\vec{i}}{\vec{i}} \underset{\sim}{*} \tilde{i} \vec{i}$ & 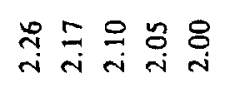 & 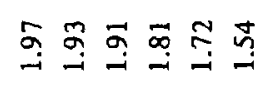 \\
\hline 유 & 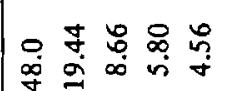 & 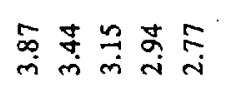 & 苔芯导导柋 & 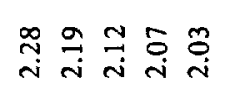 & ğ \\
\hline$\stackrel{\infty}{\rightarrow}$ & 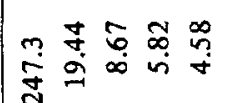 & 呑热 & 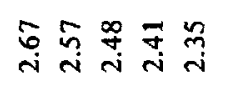 & 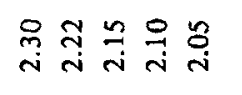 & 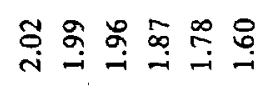 \\
\hline $\mathscr{0}$ & 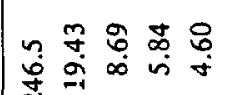 & 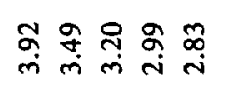 & 尽克 & $\stackrel{m}{i} \underset{i}{\stackrel{\infty}{i}} \stackrel{\infty}{i} \stackrel{0}{i}$ & 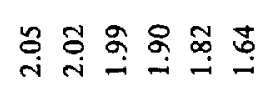 \\
\hline \pm & 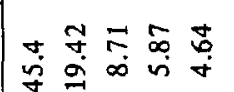 & 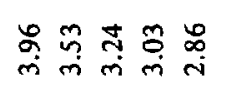 & 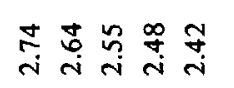 & 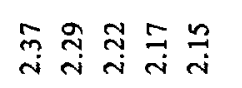 & gి \\
\hline$\simeq$ & 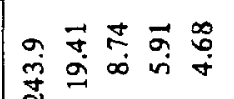 & 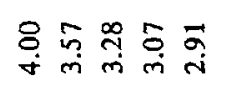 & શิ & 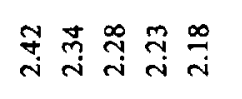 & 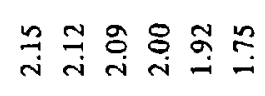 \\
\hline 오 & 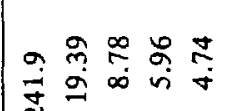 & 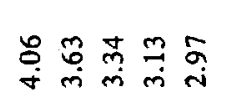 & 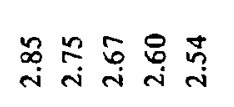 & $\underset{\sim}{\vec{i}} \vec{i} \underset{\sim}{\sim} \underset{i}{\stackrel{D}{i}} \underset{i}{\sim}$ & 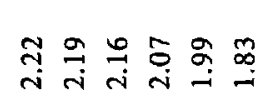 \\
\hline$a$ & 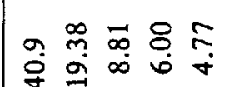 & 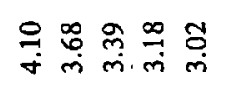 & 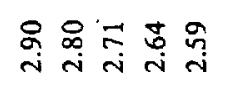 & 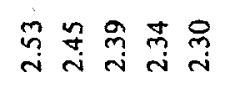 & 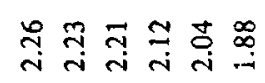 \\
\hline$\infty$ & 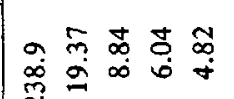 & 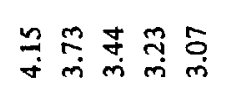 & 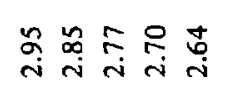 & ڤ్ & 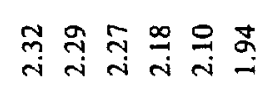 \\
\hline$r$ & 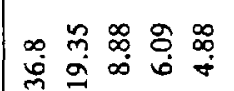 & 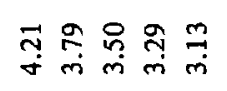 & 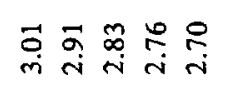 & 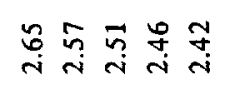 & 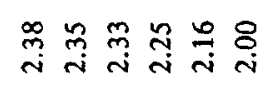 \\
\hline 0 & 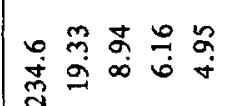 & 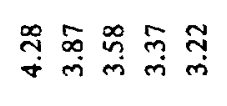 & 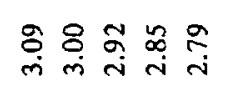 & 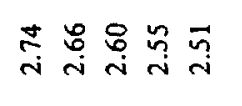 & 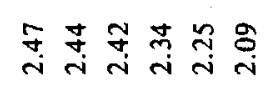 \\
\hline in & 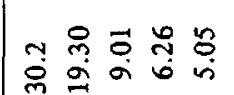 & 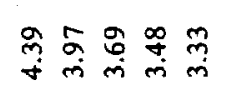 & 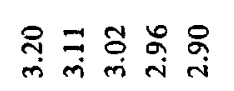 & 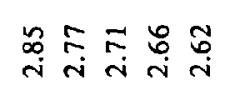 & 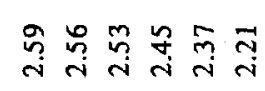 \\
\hline$\sigma$ & 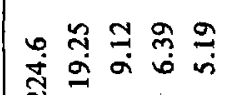 & 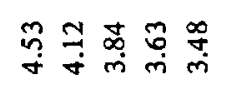 & 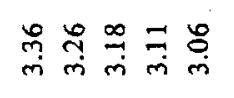 & 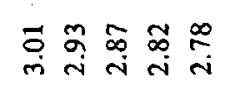 & 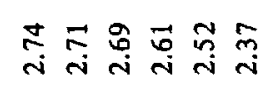 \\
\hline$m$ & 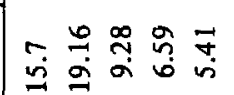 & 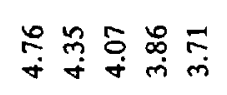 & ڤ్ర్య & 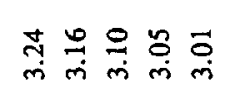 & 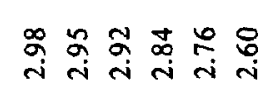 \\
\hline r & ๙ & 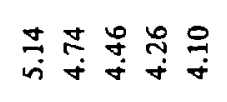 & 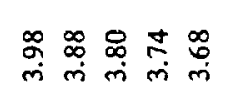 & 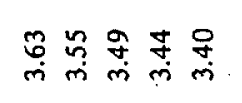 & 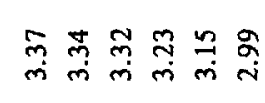 \\
\hline- & $\begin{array}{l}\vec{a} \\
\overrightarrow{0} \\
0\end{array}$ & 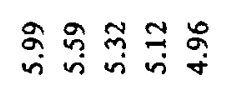 & 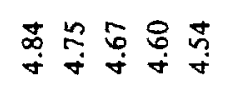 & 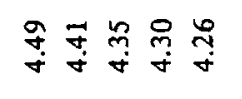 & 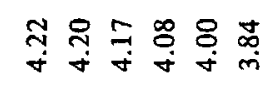 \\
\hline 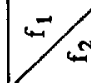 & & 0 & & 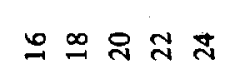 & \\
\hline
\end{tabular}




\begin{tabular}{|c|c|c|c|c|c|c|}
\hline & 8 & 总员 & 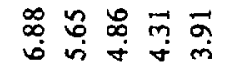 & 萹品 & 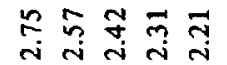 & $\begin{array}{l}m \\
\dot{i}\end{array}$ \\
\hline & $\mathbb{N}$ & 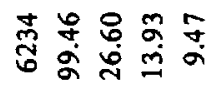 & 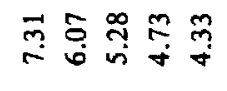 & రृ & 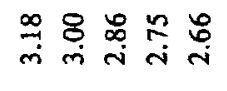 & 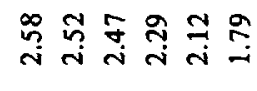 \\
\hline & $\pi$ & สี & 总寻总察尔 & 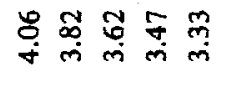 & ત્山 & 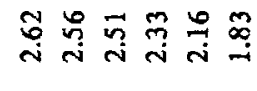 \\
\hline & ๙ & 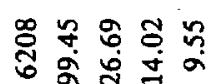 & 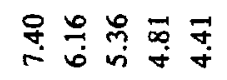 & 央 & 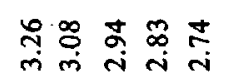 & 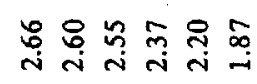 \\
\hline & $\stackrel{\infty}{\sim}$ & 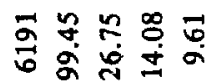 & 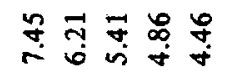 & 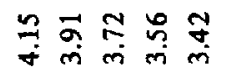 & 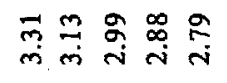 & 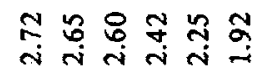 \\
\hline & $\mathscr{1}$ & 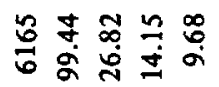 & 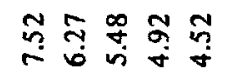 & స్ & 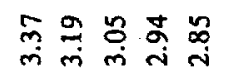 & 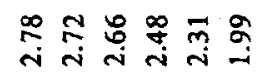 \\
\hline & \pm & 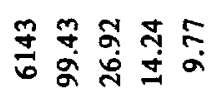 & 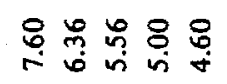 & 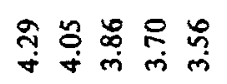 & 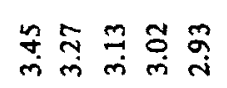 & 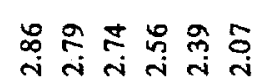 \\
\hline & $\simeq$ & 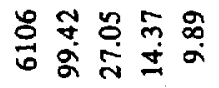 & 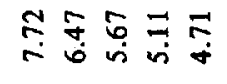 & 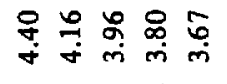 & 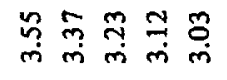 & 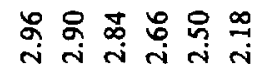 \\
\hline 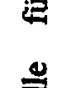 & 요 & 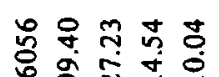 & 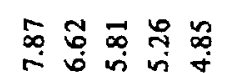 & 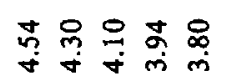 & 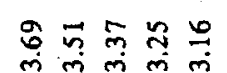 & ô \\
\hline & a & క్రి & 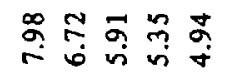 & 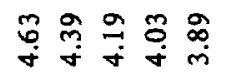 & 总 & 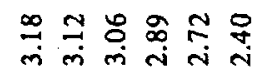 \\
\hline & $\infty$ & 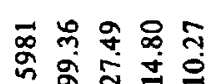 & 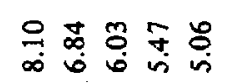 & 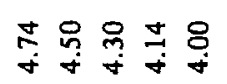 & 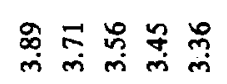 & 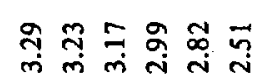 \\
\hline & $r$ & 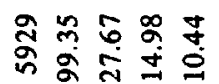 & 巡 & 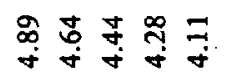 & 乫 总 & 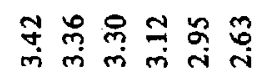 \\
\hline & 0 & 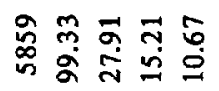 & 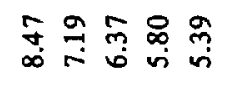 & 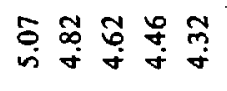 & 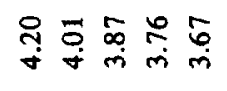 & 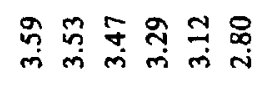 \\
\hline & $n$ & 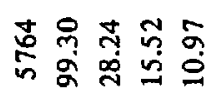 & 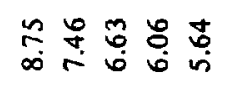 & 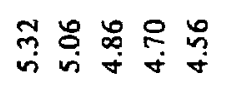 & 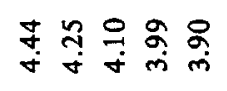 & 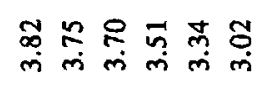 \\
\hline & + & 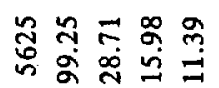 & 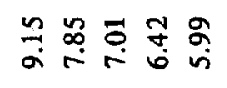 & 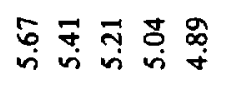 & 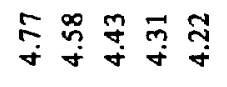 & 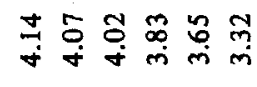 \\
\hline & $m$ & 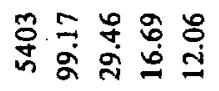 & 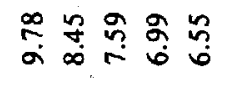 & สี่ & 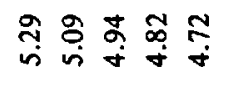 & 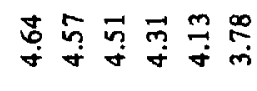 \\
\hline & $N$ & 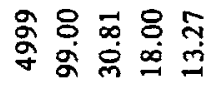 & 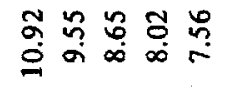 & 궁 & đु్ & 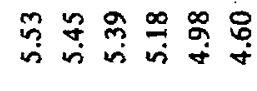 \\
\hline & - & 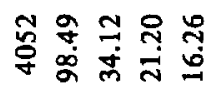 & 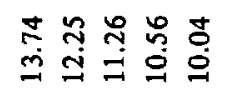 & 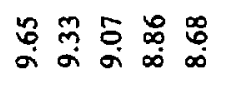 & 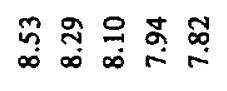 & 芯志虽蒠总志 \\
\hline & $=10$ & $\rightarrow N$ & & & $\underline{0}$ & \\
\hline
\end{tabular}




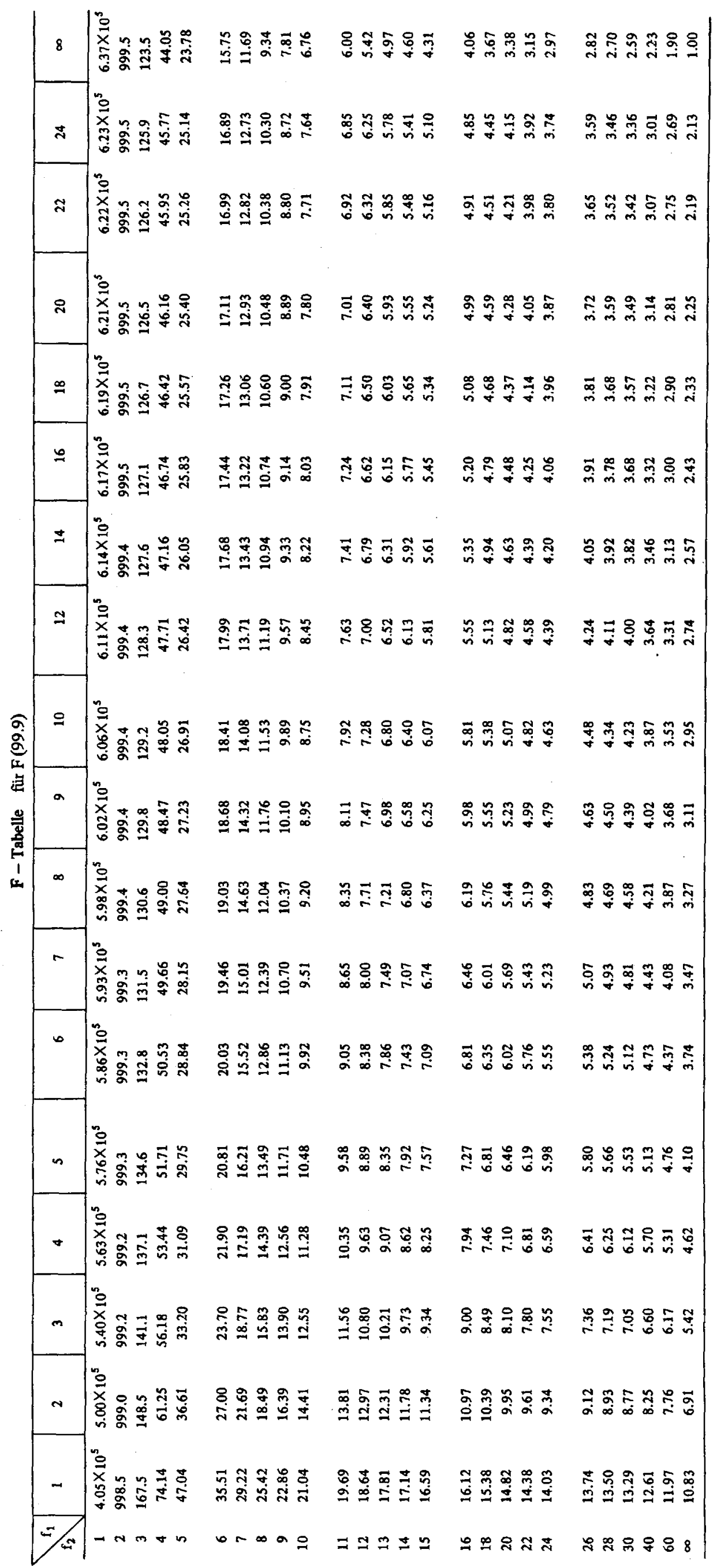




\section{Les Erreurs en Chromatographie}

\section{Erreurs fortuites (2 ${ }^{\mathrm{eme} e}$ partie)}

\section{R. Kaiser}

BASF M 325, D-67 Ludwigshafen/Rh.
You can read this paper in English language on page 215 Die gleiche Arbeit können Sie in deutscher Sprache auf Seite 220 lesen
Dans une précédente série de pages vertes concernant les erreurs, il a été établi que chaque mesure est par nature entachée d'erreur. Les résultats de mesure sont appelés données.

Des jugements ne peuvent être portés et des décisions prises à partir de ces données que si on connait:

1. la valeur moyenne $\bar{x}$ d'une série de mesures

2. l'écart-type s

3. le nombre total de mesures $\mathbf{n}$

et si on effectue sur ces grandeurs caractéristiques des tests de statistique mathématique.

Les résultats de ces tests constituent la seule base permettant, à partir des valeurs d'une mesure, de porter un jugement ou de prendre une décision qui soient scientifiquement indiscutables.

On a montré comment calculer la valeur moyenne (moyenne arithmétique) $\bar{x}$ et l'écart-type approché s et on a utilisé comme premier test la méthode de Nalimov basée sur la mise en évidence des valeurs aberrantes.

On décrira maintenant une méthode graphique de mathématique statistique qui fournira les renseignements indiqués plus loin. On vérifie d'abord que les données sont statistiquement normalement réparties. C'est seulement alors qu'il est permis d'utiliser les $t \in s t s(t, F)$ décrits ciaprès. En vérifiant que la répartition des données est normale, on obtient également les valeurs approchées de la valeur moyenne $\bar{x}$ et de l'écart-type s.

En règle générale, les valeurs numériques obtenues en chromatographie correspondent à une répartition normale. Si la méthode de vérification graphique montre que la répartition n'est pas normale, on doit alors en répétant les mesures, augmenter le nombre de données et former des groupes de valeurs moyennes. Les valeurs moyennes seront avec une probabilité encore plus grande, normalement réparties. C'est une erreur très répandue de croire que pour effectuer des tests statistiques, il est nécessaire de répéter les mesures un très grand nombre de fois.

On peut déjà, à partir de trois mesures, calculer un écarttype approché et quatre à six mesures répétées permettent d'effectuer des tests statistiques relativement précis. Il n'est pas à recommander, en chromatographie, d'effectuer plus de dix mesures si le coût d'une mesure est élevé.
Bien que normalement, il soit souhaitable de disposer d'une vingtaine de mesures, on peut déjà à partir de 3 à 10 mesures vérifier (grossièrement) que l'on a une répartition statistiquement normale.

\section{Vérification de la répartition normale dans le cas de trois à vingt mesures}

Pour la détermination graphique approchée de la valeur moyenne et de l'écart-type, il convient de procéder de la manière suivante:

1. Déterminer de trois à vingt valeurs élémentaires (exemple: cinq valeurs élémentaires provenant de la répétition d'une mesure).

2. Classer les valeurs par ordre croissant en un tableau et les numéroter en suivant de $\mathrm{a}=1$ à $\mathrm{a}=\mathrm{n}$; cette numéro tation sera nécessaire pour pouvoir calculer les ordonnées $\mathrm{y}_{1}$ à $\mathrm{y}_{\mathrm{n}}$ selon la formule ci-dessous.

3. Calculer les ordonnées $y_{1}$ à $y_{n}$ pour les valeurs élémentaires selon la formule

$y=50+100 \frac{a-\frac{n+1}{2}}{n}$

et porter la valeur de y dans un tableau (voir exemple ci-dessous).

Exemple:

Résultats de mesure: 37,$70 ; 36,28 ; 37,30 ; 37,13$; 36,88 .

Soit 5 valeurs pour la grandeur mesurée, donc $n=5$. Classer les valeurs par ordre croissant et leur attribuer un numéro d'ordre de $\mathrm{a}=1$ à $\mathrm{a}=\mathrm{n}$; calculer $\mathrm{y}$ :

\begin{tabular}{c|c|c}
\hline numéro d'ordre & Résultats & Ordonnées \\
\hline $\mathrm{a}=1$ & 36.28 & $\mathrm{y}_{1}=10$ \\
2 & 36.88 & $\mathrm{y}_{2}=30$ \\
3 & 37.13 & $\mathrm{y}_{3}=50$ \\
4 & 37.30 & $\mathrm{y}_{4}=70$ \\
$5=\mathbf{n}$ & 37.70 & $\mathrm{y}_{5}=90$ \\
\hline
\end{tabular}

Exemple de calcul de l'ordonnée $y_{2}$ pour le deuxième résultat (à $a=2$ correspond $x_{2}=36,88$ ):

formule: $y_{2}=50+100 \frac{a-\frac{n+1}{2}}{n}$ 
valeurs: $\quad a=2 ; n=5$

$$
\text { calcul: } \quad \begin{aligned}
y_{2} & =50+100 \frac{2-\frac{5+1}{2}}{5} \\
& =50+100 \frac{2-3}{5} \\
& =50+100\left(-\frac{1}{5}\right) \\
& =50-20 \\
y_{2} & =30
\end{aligned}
$$

4. Prendre un diagramme de probabilité ayant en abscisse une échelle linéaire et en ordonnée une échelle correspondant à l'intégrale de Gauss. Porter sur le papier graphique les valeurs mesurées $\left(\mathrm{x}_{1}\right.$, etc. ..) en fonction des valeurs calculées de $y$. Désigner par $g$ l'axe des $x$, par $\Sigma \%$ l'axe des y. Joindre par une droite les points ainsi déterminés: c'est la droite dite de Hazen.

A la valeur d'ordonnée $y=50 \%$ correspond la valeur d'abscisse $\bar{x}$, ou valeur moyenne des cinq mesures. Aux valeurs d'ordonnée $y=15,9 \%$ et $y=84,1 \%$ correspondent deux valeurs d'abscisses qui marquent les valeurs limite $-s$ et $+s$ correspondant à l'écarttype approché. On peut lire sur la figure 1:

$\overline{\mathbf{x}}$ se situe à $\sim 37,05$

- $s$ se situe à 36,51

$+s$ se situe à 37,60

La distance entre $(-s)$ et $(+s)$ égale à $2 s$ est numériquement égale à $37,60-36,51=1,09$. On a donc $2 \mathrm{~s}=1,09$ et l'écart-type approché $s=0,55$.
Si on calcule $\overline{\mathrm{x}}$ et $\mathrm{s}$ d'après les formules données dans la première série de pages vertes, on obtient:

$$
\begin{aligned}
\bar{x} & =37,058 \\
\mathrm{~s} & =0,53
\end{aligned}
$$

Il est bien clair que la méthode que l'on vient de décrire conduit à des données très approximatives qui sont à leur tour entachées d'erreurs. Il est par conséquent souhaitable d'introduire un plus grand nombre de données pour vérifier que leur répartition est statistiquement normale. Si on a vingt valeurs élémentaires ou davantage, on peut procéder de la manière suivante:

1. Etablir la différence existant entre le résultat de mesure le plus élevé et le résultat de mesure le plus faible

$\begin{array}{lllll}\text { exemple: } & 36.6 & 38.3 & 37.4 & 35.4 \\ & 38.5 & 37.2 & 36.7 & 37.6 \\ & 35.1 & 36.6 & 36.1 & 39.5 \\ & 37.3 & 38.0 & 37.1 & 35.1 \\ & 36.4 & 38.7 & 36.5 & 37.0\end{array}$

valeur maximale: 39.5

valeur minimale: 35.1

Différence: $\quad 4.4$

2. Répartir tous les résultats de mesure en cinq à six domaines de valeurs d'égale importance. Dans l'exemple précédent il apparait que cette règle est satisfaite par l'établissement de cinq domaines de 35,0-36,0; $36,0-37,0 ; 37,0-38,0 ; 38,0-39,0$ et $39,0-40,0$ puisque la différence entre les valeurs maximale et minimale s'élève à $4,4 \sim 5$.

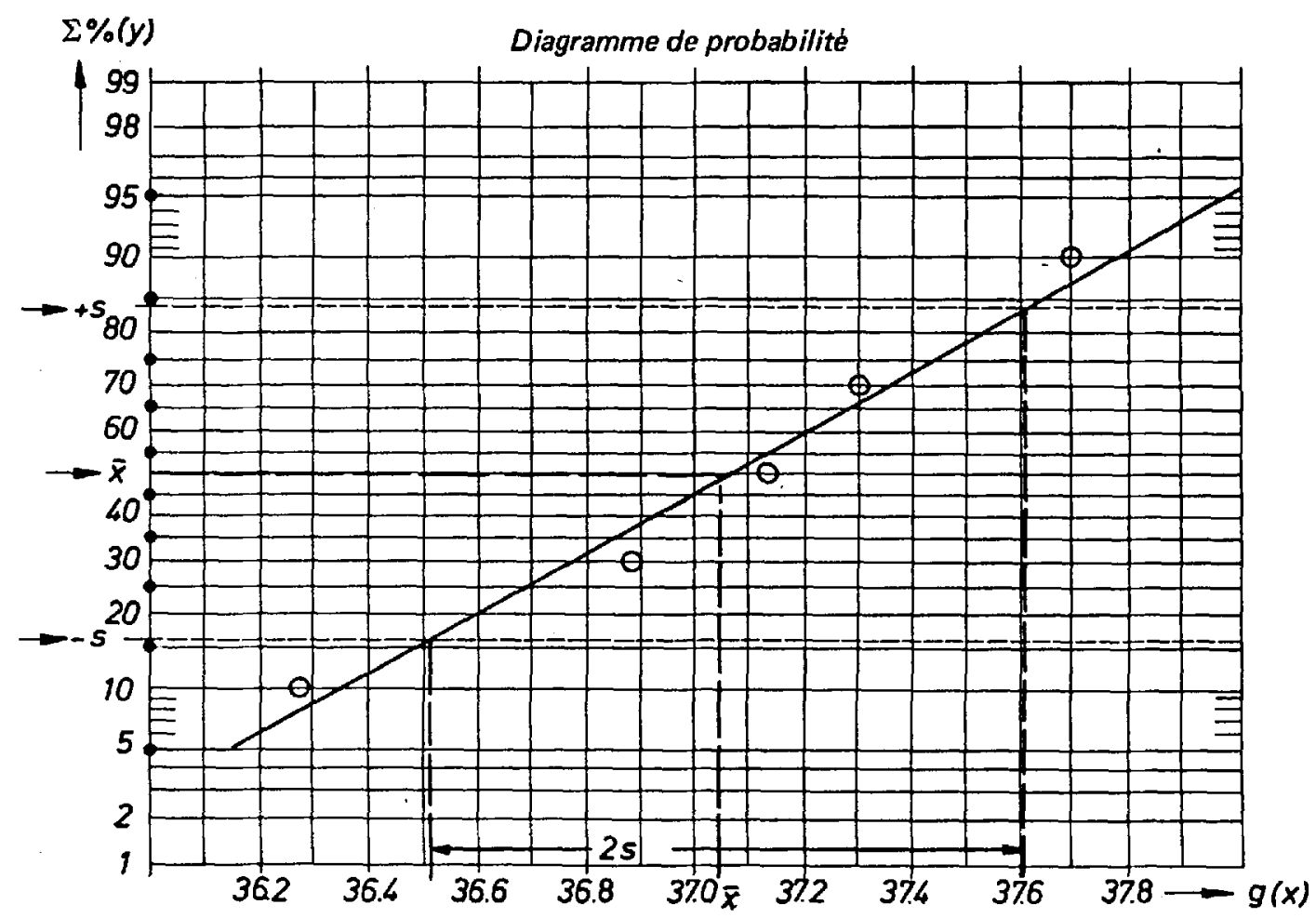

Fig. 1 
3. Noter le nombre de résultats appartenant à chaque domaine, compter les résultats tombant exactement à la limite de deux domaines comme appartenant par moitié au domaine supérieur et par moitié au domaine inférieur.

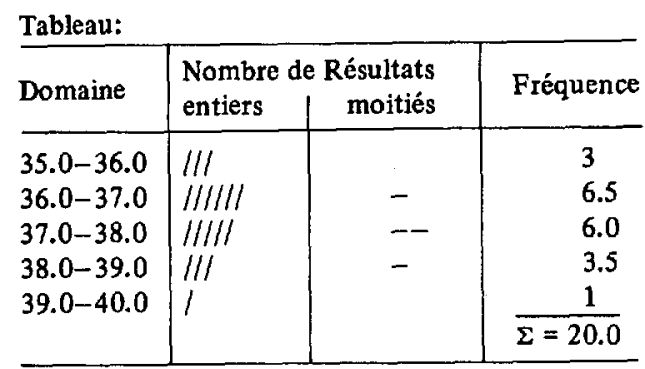

4. Exprimer en pourcentage partiel et total la fréquence d'apparition des valeurs dans chaque domaine.

Exemple: Le nombre de résultats est égal à 20 . Dans le domaine $39,0-40,0$ se trouve un résultat, soit $5 \%$ des 20 mesures.

\begin{tabular}{l|c|l|c}
\hline Domaine & Fréquence & Fréquence \% & $\begin{array}{c}\text { Totaux partiels \% } \\
(\Sigma \%)\end{array}$ \\
\hline $35.0-36.0$ & 3 & $=15.0 \%$ & $15.0 \%$ \\
$36.0-37.0$ & 6.5 & $=32.5 \%$ & $47.5 \%$ \\
$37.0-38.0$ & 6.0 & $=30.0 \%$ & $77.5 \%$ \\
$38.0-39.0$ & 3.5 & $=17.5 \%$ & $95.0 \%$ \\
$39.0-40.0$ & 1 & $=5.0 \%$ & $100.0 \%$ \\
\hline
\end{tabular}

5. Porter sur un diagramme de probabilité ayant en abscisse une échelle linéaire et en ordonnée une échelle correspondant à l'intégrale de Gauss, les points ayant pour coordonnées les valeurs $\Sigma \%(15,0 \%, 47,5 \%$, $77,5 \%$, etc. . .) et la limite supérieure du domaine correspondant $(\mathrm{g})$, ainsi pour $\mathrm{x}=36,0, \Sigma \%=15,0$, pour $\mathrm{x}=37,0, \Sigma \%=47,5$, pour $\mathrm{x}=38,0, \Sigma \%=77,5$ etc. . . (voir figure 2).

A la valeur d'ordonnée $\Sigma \%=50$ correspond la valeur d'abscisse $\mathrm{x}=\overline{\mathbf{x}}$, c'est à dire la valeur moyenne des 20 mesures.

Entre les valeurs d'abscisse correspondant à $\Sigma \%=15,9$ et à $\Sigma \%=84,1$ se trouve le domaine de " $-s$ à $+s$ " égal à $2 \mathrm{~s}$.

On lit sur la figure $2 \quad \bar{x}=37,11$

2 s est égal à $38,28-35,96=2,32$ donc

$2 \mathrm{~s}=2,32$ et $\mathrm{s}=1,16$.

Le calcul numérique donne:

$\overline{\mathrm{x}}=37,055$

$s=1,1763$

L'étude graphique des valeurs numériques a montré que la dispersion des résultats de mesure correspond à une répar. tition normale et que les points obtenus s'alignent bien sur une droite. Par conséquent, le procédé de mesure donne des résultats normalement répartis et les tests statistiques suivants peuvent être effectués.

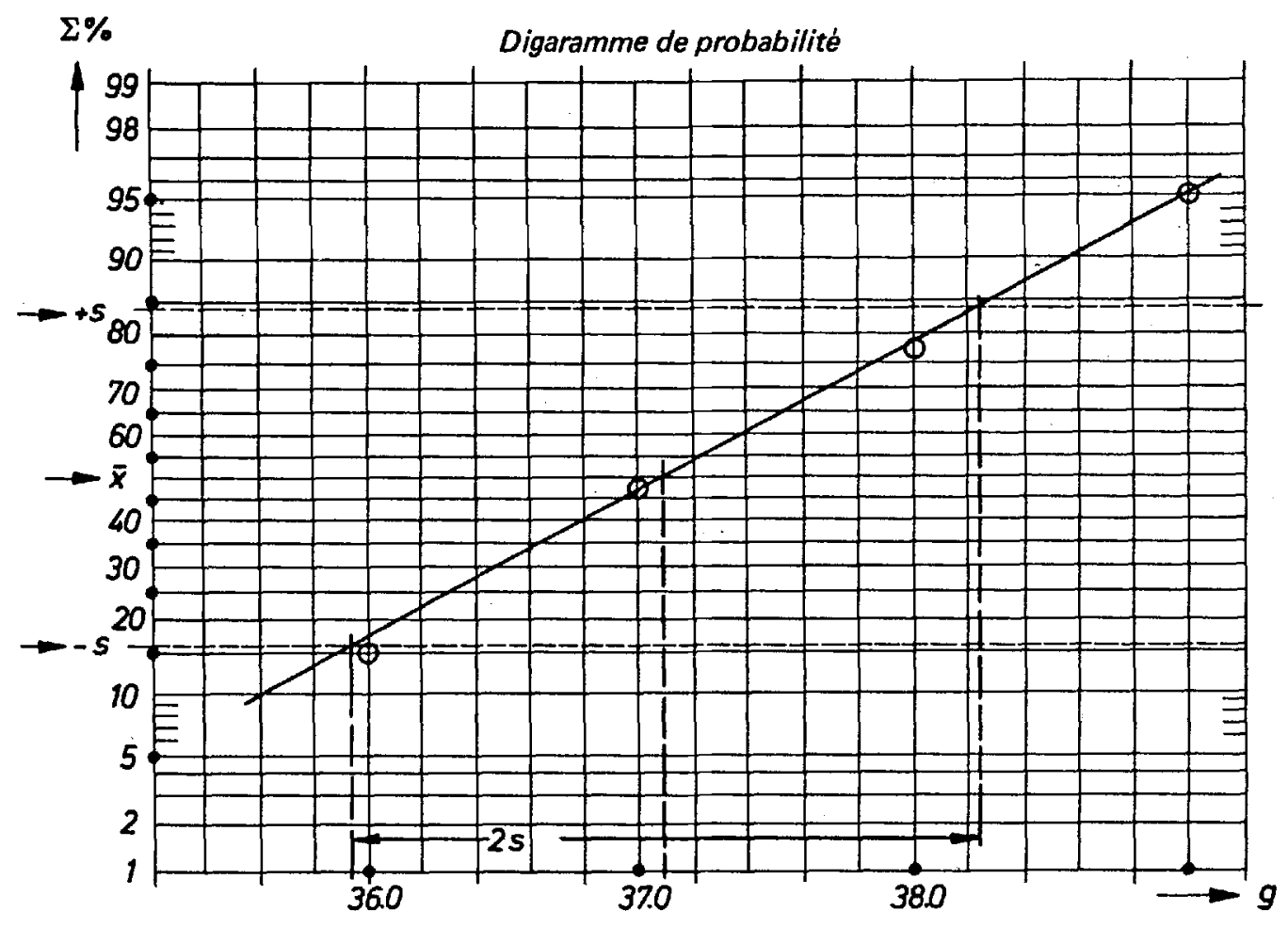

Fig. 2 


\section{Test " $t_{\Downarrow}$ pour la comparaison des valeurs moyennes}

Ce test permet de dire si deux valeurs moyennes $\bar{x}_{A}$ et $\bar{x}_{B}$ diffèrent réelement l'une de l'autre ou si leur différence est dépourvue de signification.

Une telle appréciation basée sur des faits de mathématique statistique permet de tirer les conclusions suivantes.

a) si deux échantillons ont été comparés et que des erreurs de mesure systématiques sont exclues: les échantillons ayant servi aux mesures sont différents

b) si deux échantillons exactement semblables ont été examinés dans deux appareils ou deux systèmes de séparation: l'un des deux ou les deux appareils ou systèmes de séparation donne (ou donnent) une erreur de mesure systématique (ceci vaut aussi pour la comparaison de détecteurs, intégrateurs, facteurs d'étalonnage, etc...).

c) si $\bar{x}_{A}$ et $\bar{x}_{B}$ sont le résultat de mesures faites sur le même produit à des moments ou en des lieux différents: on doit en faire la moyenne, à condition que le test $F$ de comparaison des écart-type le permette.

A On a besoin des données $\bar{x}_{A}, s_{A}, n_{A}$ et $\bar{x}_{B}, s_{B}, n_{B}$.

B On calcule la valeur d'essai $\tau$

$$
\begin{aligned}
& \tau=\left|\frac{\bar{x}_{A}-\bar{x}_{B}}{s_{d}}\right| \times \sqrt{\frac{n_{A} \times n_{B}}{n_{A}+n_{B}}} \\
& s_{d}=\sqrt{\frac{\left(n_{A}-1\right) \times s_{A}^{2}+\left(n_{B}-1\right) \times s_{B}^{2}}{n_{A}+n_{B}-2}}
\end{aligned}
$$

C On compare $\tau$ aux valeurs statistiques $t(95), t(99)$ et $t(99,9)$ qui dépendent de $f=n_{A}+n_{B}-2$. On trouve dans le tableau « $t$ » (voir ci-dessous) les valeurs statistiques $t(95 . .99,9)$ en fonction de $f$.

Si $\tau \geqslant t(95), \bar{x}_{\mathrm{A}}$ ne diffère pas de $\overline{\mathrm{x}}_{\mathrm{B}}$. On doit tirer des deux valeurs une moyenne d'ensemble $\overline{\bar{x}}$, ce qui présente l'avantage de rendre possibles des tests statistiques ultérieurs d'une plus grande précision puisque $n$ est alors la somme de $n_{A}$ et $n_{B}$. Dans la prochaine série de pages vertes on indiquera comment calculer simultanément «l'écart-type commun» et la «valeur moyenne d'ensemble» pour tous les résultats de mesure de $\overline{\mathrm{x}}_{\mathrm{A}}$ et $\overline{\mathrm{x}}_{\mathrm{B}}$.

Si $t(95) \leqslant \tau<t(99): \bar{x}_{A}$ est, avec une probabilité de $95 \%$, différent de $\overline{\mathrm{x}}_{\mathrm{B}}$.

Si $t(99) \leqslant \tau<t(99,9): \bar{x}_{A}$ est, d'une manière significative différent de $\overline{\mathrm{x}}_{\mathrm{B}}$.

Si $\tau \geqslant t(99,9), \bar{x}_{A}$ est, d'une manière hautement significative, différent de $\bar{x}_{B}$

\section{Exemple numérique}

$$
\begin{aligned}
& \mathrm{n}_{\mathrm{A}}=5 \quad \overline{\mathrm{x}}_{\mathrm{A}}=30.445 \quad \mathrm{~s}_{\mathrm{A}}= \pm 0.193 \\
& \mathrm{n}_{\mathrm{B}}=6 \quad \overline{\mathrm{x}}_{\mathrm{B}}=30.611 \quad s_{\mathrm{B}}= \pm 0.169 \\
& s_{d}=\sqrt{\frac{4 \times 0.193^{2}+5 \times 0.169^{2}}{5+6-2}}=0.18006 \\
& \tau=\left|\frac{30.445-30.611}{0.18006}\right| \times \sqrt{\frac{5 \times 6}{5+6}}=1.522 \\
& n_{A}=5 \text { et } n_{B}=6 \text {, donc } f=5+6-2=9
\end{aligned}
$$

Pour $f=9$, on trouve dans le tableau $t$ :

$$
t(95)=2.26
$$

$\mathrm{t}(99)=3.25$

$\mathrm{t}(99.9)=4.78$

Conclusion: $\tau=1,522$ est infêrieur à $\mathrm{t}(95)=2,26$ donc les valeurs moyennes $\overline{\mathrm{x}}_{\mathrm{A}}$ et $\overline{\mathrm{x}}_{\mathrm{B}}$ ne sont pas différentes.

C'est le test $\mathrm{F}$ qui permettra de dire si on peut en tirer une nouvelle valeur moyenne.

\section{Test $~ " F_{》}$ pour la comparaison des écarts-type}

Ce test permet de dire si deux écarts-type $s_{A}$ et $s_{B}$ diffèrent nettement l'un de l'autre ou si leur différence n'est pas significative. Une telle évaluation, basée sur des données de statistique mathématique permet de tirer les conclusions suivantes:

a) lorsque les données proviennent de deux instruments, de deux procédés de mesure, de deux méthodes de détermination ou ont été obtenues par deux opérateurs, on peut dire si les procédés, les appareillages ou les méthodes de travail diffèrent d'une manière significative. Le meilleur procédé, le meilleur appareil ou la meilleure méthode de travail est celui (ou celle) qui, toutes conditions égales par ailleurs (même échantillon) donne l'écart-type le plus faible.

b) si, pour deux séries de mesures, les écarts-type ne diffèrent pas d'une manière significative et que, selon le test $t$ (voir ci-dessus), les valeurs moyennes ne diffèrent pas on peut utiliser tous les résultats des deux séries de mesures pour en établir la moyenne

A On a besoin de connaître les données $s_{A}, n_{A}, s_{B}$ et $n_{B}$

B On calcule la valeur d'essai « $P F$ »

$$
\mathrm{PF}=\left(\frac{s_{A}}{s_{B}}\right)^{2}, \text { si } s_{A}>s_{B}
$$

ou

$$
\mathrm{PF}=\left(\frac{\mathrm{s}_{\mathrm{B}}}{\mathrm{s}_{\mathrm{A}}}\right)^{2}, \text { si } \mathrm{s}_{\mathrm{B}}>\mathrm{s}_{\mathrm{A}} \text {. }
$$

On attribue l'indice 1 au terme $\mathrm{n}$ de chaque groupe de données qui correspond à l'écart-type maximal. Ainsi si $s_{B}>s_{A}, n_{B}=n_{1}$ et $n_{A}=n_{2}$.

C On compare PF aux valeurs statistiques $F(95), F(99)$ et $F(99,9)$ pour $f_{1}=n_{1}-1$ et $f_{2}=n_{2}-1$. On trouvera au tableau $F$ les valeurs statistiques $F(95, \ldots, 99,9)$ en fonction de $f_{1}$ et $f_{2}$.

Si $P F \geqslant F(95), s_{A}$ et $s_{B}$ ne diffèrent pas. On peut, siselon le test $t$ (voir ci-dessus) - les valeurs moyennes $\overline{\mathrm{x}}_{\mathrm{A}}$ et $\overline{\mathrm{x}}_{\mathrm{B}}$ ne diffèrent pas non plus de manière significative, calculer à partir de la totalité des données un nouveau groupe de données $\overline{\bar{x}}, \overline{\bar{s}}$ et $\overline{\overline{\mathrm{n}}}$, statistiquement encore plus précises. Ceci sera décrit dans la prochaine série de pages vertes.

Si $F(95) \leqslant P F<F(99): s_{A}$ diffère de $s_{B}$ avec une probabilité de $95 \%$.

Si $F(99) \leqslant P F<F(99,9): s_{A}$ diffère de $s_{B}$ d'une manière significative.

Si $P F \geqslant F(99,9): s_{A}$ diffère de $s_{B}$ d'une manière hautement significative 


\section{Exemple numérique}

$$
\begin{array}{ll}
\mathrm{n}_{\mathrm{A}}=4 & \mathrm{~s}_{\mathrm{A}}=0.2525 \\
\mathrm{n}_{\mathrm{B}}=5 & \mathrm{~s}_{\mathrm{B}}=0.0856 \\
\mathrm{PF}=\left(\frac{0.2525}{0.0856}\right)^{2}=8.701 \\
\mathrm{n}_{\mathrm{A}}=\mathrm{n}_{1}, \text { puisque } & \mathrm{s}_{\mathrm{A}}>\mathrm{s}_{\mathrm{B}} \\
\mathrm{f}_{1}=\mathrm{n}_{\mathrm{A}}-1, & \mathrm{f}_{2}=\mathrm{n}_{\mathrm{B}}-1 \\
\mathrm{f}_{1}=3 \quad, & \mathrm{f}_{2}=4
\end{array}
$$

Pour ces valeurs de $f_{1}$ et $f_{2}$, on a: $F(95)=6.59$

$F(99)=16.69$

$F(99.9)=56.18$

Donc: $F(99)>P F=8.701>F(95)$, par conséquent $\mathrm{s}_{\mathrm{A}}$ est vraisemblablement supérieur à $\mathrm{s}_{\mathrm{B}}$.

Si on n'est pas satisfait de ce résultat ne donnant qu'une probabilité de $95 \%$, on doit procéder à un plus grand nombre de mesures. Le test $F$ n'est pas particulièrement précis quand on ne dispose que d'un trop petit nombre de mesures.

Tableau «t»: voir page 224

Tableaux «F»: voir pages $225,226,227$ 